\title{
On the Metastability of the 1-d Allen-Cahn Equation
}

\author{
Maria G. Westdickenberg ${ }^{1}$
}

Received: 30 October 2019 / Revised: 28 May 2020 / Published online: 27 July 2020

(c) The Author(s) 2020

\begin{abstract}
We apply an energy method for metastability, developed in an earlier work with Otto, to the Allen-Cahn equation on the line and a broad class of initial data. In the earlier work, we for simplicity considered the equation on $(0, L) \times(0, \infty)$ with periodic boundary conditions and an initial condition with two simple zeros. In this paper we explain the implications of the metastability framework (slightly modified as in previous joint work with Scholtes) for the equation on $\mathbb{R} \times(0, \infty)$ and a more general initial condition. Our goal is to make clear the strength of the metastability framework and to highlight the difference in the analysis between the second-order Allen-Cahn equation and the fourth-order Cahn-Hilliard equation.
\end{abstract}

Keywords Energy-energy-dissipation · Nonlinear PDE · Gradient flow · Dynamic metastability

Mathematics Subject Classification 35K55 - 49N99

\section{Introduction}

Dynamic metastability refers to problems in which generic solutions spend a long time near a configuration that is not a stationary solution and eventually change dramatically. In other words, dynamically metastable systems exhibit states that appear to be stable but are not. Metastable behavior in the one-dimensional Allen-Cahn equation was pointed out by John Neu in unpublished notes and analyzed in the seminal work of Carr and Pego [10] and Fusco and Hale [14]. It has since been studied by many authors; we refer for instance to $[8,11,17,22]$ and the references therein. See also Sect. 1.4, below. A related, fourth-order equation for which metastability has been numerically and analytically captured is the one-dimensional Cahn-Hilliard equation; see for instance [2-4,7,13,15,20,21].

Here we apply the metastability framework developed in [17] to the Allen-Cahn equation on $\mathbb{R}$ in order to give a concrete analysis for generic initial conditions. (The analogous result holds on $(0, L)$ with periodic, Neumann, or Dirichlet boundary conditions.) This introduc-

$凶 \quad$ Maria G. Westdickenberg

maria@math1.rwth-aachen.de

1 RWTH Aachen University, Aachen, Germany 
tion also summarizes the differences between the two equations and the state of the art on relaxation and metastability for both equations.

The one-dimensional Allen-Cahn and Cahn-Hilliard equations are

$$
\begin{aligned}
u_{t} & =u_{x x}-G^{\prime}(u) \\
\text { and } u_{t} & =-\left(u_{x x}-G^{\prime}(u)\right)_{x x},
\end{aligned}
$$

respectively. Here $G(u)$ is a double-well potential with nondegenerate minima at \pm 1 ; a canonical choice is $G(u)=\frac{1}{4}\left(1-u^{2}\right)^{2}$ and the general assumptions are listed in Remark 1.1. Both (1.1) and (1.2) are often studied with a small parameter $\varepsilon$ appearing in the equation, in which case typical transition layers connecting 1 and -1 occur on scale $\varepsilon$. This is equivalent via rescaling to studying (1.1) and (1.2), in which transition layers are order one.

Both equations were suggested as phenomenological models for systems in which two "pure" phases \pm 1 coexist, and the value of the order parameter $u$ indicates the proportion of each phase. While the Cahn-Hilliard equation preserves the integral of the solution, the Allen-Cahn equation does not. Equations (1.1) and (1.2) are gradient flows of the scalar Ginzburg-Landau energy

$$
E(u):=\int_{\mathbb{R}} \frac{1}{2} u_{x}^{2}+G(u) d x
$$

with respect to the $L^{2}$ and $\dot{H}^{-1}$ metrics, respectively.

Both equations are also degenerate in the one-dimensional case. The degeneracy arises because the minimizer of the energy on $\mathbb{R}$ subject to \pm 1 boundary conditions is invariant under shifts; that is, there is not a unique minimizer, but an infinite family of minimizers. This degeneracy leads to metastable behavior on both the real line and large but bounded domains. Hence, the one-dimensional setting is a natural starting point in which to understand the roots and nature of metastability.

The expected behavior for both equations is the following: An initial condition that features both large regions where $u_{0}>0$ and large regions where $u_{0}<0$ will quickly relax to a configuration of large regions of $u \approx 1$ and large regions of $u \approx-1$ with so-called transition layers connecting them. Moreover the transition layers are well-approximated, up to translations and reflection, by the function $v_{1}$ satisfying

$$
-v_{1 x x}+G^{\prime}\left(v_{1}\right)=0, \quad v_{1}( \pm \infty)= \pm 1, \quad v_{1}(0)=0 .
$$

For the canonical potential, $v_{1}(x)=\tanh \left(\frac{x}{\sqrt{2}}\right)$. The function $v_{1}$ minimizes the energy $E$ subject to its boundary conditions. For future reference, we set

$$
E\left(v_{1}\right)=: e_{*} .
$$

We will refer to the approximately optimal transition layers connecting phases of \pm 1 as "kinks." These well-separated kinks slowly attract each other until all of the kinks-or as many as permitted depending on the equation and the boundary and initial conditions — collide and annihilate.

It is instructive to consider the linearization of the Allen-Cahn and Cahn-Hilliard equations on $\mathbb{R}$ around $v_{1}$, i.e.,

$$
f_{t}=f_{x x}-G^{\prime \prime}\left(v_{1}\right) f=: \mathcal{L}_{A C} f \text { and } f_{t}=-\partial_{x x}\left(f_{x x}-G^{\prime \prime}\left(v_{1}\right) f\right)=: \mathcal{L}_{C H} f .
$$

Both $\mathcal{L}_{A C}$ and $\mathcal{L}_{C H}$ have a zero eigenvalue with eigenfunction $v_{1, x}$ (corresponding to translations). While for $\mathcal{L}_{A C}$ the next eigenvalue is negative, for $\mathcal{L}_{C H}$ the essential spectrum extends all the way to zero; in other words $\mathcal{L}_{A C}$ possesses a spectral gap while $\mathcal{L}_{C H}$ does not. 
In terms of relaxation of the initial energy gap in time, a spectral gap should imply exponential-in-time convergence, and the absence of a spectral gap should mean that at most algebraic-in-time convergence can be expected. Our goal is to develop natural nonlinear estimates and show how these can be used to establish convergence results for a wide class of initial data, i.e., initial data that is not in a small neighborhood of $v_{1}$ or, more generally, the slow manifold. We will make these ideas more concrete after first reviewing the classical facts.

\subsection{Classical Exponential- and Algebraic-in-Time Relaxation to Steady State}

Consider the abstract gradient flow

$$
\begin{cases}u_{t}=-\nabla E(u) & \text { for } t>0 \\ u=u_{0} & \text { at } t=0\end{cases}
$$

Moreover let

$$
-\frac{d}{d t} E(u)=\|\nabla E(u)\|^{2}=: D(u)
$$

denote the associated dissipation. Here $\|\cdot\|$ represents the natural norm induced by the metric that is used to define the gradient in (1.5). It is a well-known and elementary fact that if $u$ converges to a given state $u_{*}$ for $t \rightarrow \infty$ and if $E$ is strictly convex (at least in a neighborhood of $u_{*}$ ), then a so-called "Energy-Energy-Dissipation" (EED) estimate of the form

$$
\frac{1}{C}\left\|u-u_{*}\right\|^{2} \leq E(u)-E\left(u_{*}\right) \leq C\|\nabla E(u)\|^{2}
$$

holds, which in turn implies that the energy gap and the distance to $u_{*}$ relax exponentially in time with rate $C$ :

$$
\frac{1}{C}\left\|u-u_{*}\right\|^{2}+E(u)-E\left(u_{*}\right) \leq 2 \exp \left(-\frac{t}{C}\right)\left(E\left(u_{0}\right)-E\left(u_{*}\right)\right) .
$$

Indeed, this fact follows easily from the "algebraic information" contained in (1.6) together with the differential information

$$
-\frac{d}{d t}\left(E(u)-E\left(u_{*}\right)\right)=\|\nabla E(u)\|^{2} \quad\left(\text { since } E\left(u_{*}\right) \text { is a constant }\right) .
$$

An observation of Brezis [5] is that a gradient flow of the form (1.5) for an energy $E$ that is convex but not strictly convex satisfies the interpolation estimate

$$
E(u)-E\left(u_{*}\right) \leq \sqrt{H D},
$$

where $H:=\left\|u-u_{*}\right\|^{2}$ represents the squared distance to the equilibrium state. At the same time, Brezis points out that, in addition to (1.8), one has the differential relationships

$$
\frac{d}{d t} H \leq 0, \quad \frac{d}{d t} D \leq 0
$$

From this algebraic and differential information, one can again in an elementary way deduce

$$
H \leq H_{0}, \quad E(u)-E\left(u_{*}\right) \leq \min \left\{E\left(u_{0}\right)-E\left(u_{*}\right), \frac{H_{0}}{t}\right\}, \quad D \leq \frac{4 H_{0}}{t^{2}} .
$$

Hence an energy that is convex but not strictly convex satisfies (merely) algebraic-in-time relaxation with rate $t^{-1}$. 


\subsection{Not Convex, But Close Enough}

Both the Allen-Cahn and Cahn-Hilliard equations are interesting precisely because of their nonconvexity; they are the prototypical examples of equations exhibiting the metastable behavior of being "stuck" near states that are far from any long-time limit.

In analogy to (1.6), the EED estimates from [17] are a way to capture convexity of the energy gap transverse to the slow manifold. However (1.8) fails to hold in this case: The energy is not constant on the slow manifold, but merely constant up to small error terms. Hence the metastability framework from [17] expresses that a relation like (1.6) together with constancy of the energy on the slow manifold up to small error terms can be used to deduce dynamic metastability and, in particular, exponential-in-time relaxation of the energy gap and distance to the slow manifold (with the sharp constant in the exponential).

For the Cahn-Hilliard equation on the line, the absence of a spectral gap means that no relation like (1.6) can hold. For the Cahn-Hilliard equation on a bounded domain $(0, L)$, the Poincaré inequality delivers an $L$-dependent EED relation (morally speaking, a relation like (1.6) with $C$ replaced by $C L^{2}$ ). However, as explained in [20], in the absence of a better ( $L$ independent) relaxation estimate, this only suffices to prove metastability for well-prepared initial data.

In order to obtain a better result for the Cahn-Hilliard equation on $(0, L)$, i.e., to be able to handle generic initial data, the idea is that, absent a strict convexity condition like (1.6), one should try to establish a kind of non-strict convexity along the lines of (1.9) - at least up to higher order error terms. The paper [19] carries this out, showing that the Cahn-Hilliard equation on the line indeed satisfies mild perturbations of (1.9) and (1.10), and deduces from this information the algebraic-in-time relaxation of the energy gap

$$
E(u)-E\left(v_{1}\right) \lesssim \frac{H_{0}+E\left(u_{0}\right)-E\left(v_{1}\right)}{t} .
$$

Here $v_{1}$ is the kink from (1.4) and $H_{0}$ denotes the initial squared $\dot{H}^{-1}$ distance to $v_{1}$, which is assumed to be finite (but not small). We remark that subsequent work [18] treats $L^{1}$ - (rather than $\dot{H}^{-1}$ ) disturbances.

The $\frac{1}{t}$ relaxation from [19] is used in [20] to show that a broad class of initial data relax into a small neighborhood of the slow manifold and hence establish (exponentially long) metastability for the Cahn-Hilliard equation on a bounded domain and initial data that do not satisfy a well-preparedness condition.

Such a complex argument should not be-and indeed, is not-necessary for the AllenCahn equation on either bounded or unbounded domains. Given the preceding discussion, there should be EED estimates for the Allen-Cahn equation that imply exponential-in-time relaxation of the energy gap and metastability for a wide class of initial data. The point of this paper is to make this statement precise.

While the essential ingredients for the proofs can be found in $[17,20]$, some modification is necessary and it seems worthwhile to state the strongest possible result and gather the necessary ingredients in one place. Our result appears below in Theorem 1.8. Even for longtime convergence, the situation for Allen-Cahn is more complicated than in (1.6), since there is a whole continuum of minimizers (an infinite dimensional example of a Mexican-hat type potential); we summarize the long-time limit in Theorem 1.11. 


\subsection{Setting and Result}

We consider the Allen-Cahn equation (1.1) on $\mathbb{R}$. The analogous result can be stated for a bounded domain with periodic, Neumann, or Dirichlet boundary conditions; the adjustments are not difficult and we leave the details to the reader.

Remark 1.1 For the potential $G$ we assume:

- $G$ is $C^{2}$ and even,

- $G(u)>0$ for $u \neq \pm 1$ and $G( \pm 1)=0$,

- $G^{\prime}(u) \leq 0$ for $u \in[0,1]$ and $G^{\prime \prime}( \pm 1)>0$.

The assumption that $G$ is even is made for conceptual simplicity alone and may be relaxed.

We will now explain the set that we will use to represent the slow manifold of the evolution. For a number $N \in \mathbb{N}$ and a lengthscale $\ell \in(0, \infty)$, the collection of so-called alternating-sign energy-optimal profiles with $N$ zeros and separation distance $\ell$ is the set:

$\mathcal{N}_{N}(\ell):=\left\{v \in-1+H^{1}(\mathbb{R})\right.$ with $N$ simple zeros $x_{1}, \ldots, x_{N}$, such that $\left|x_{i+1}-x_{i}\right| \geq \ell$, $v$ minimizes the energy on $\left(x_{i}, x_{i+1}\right)$ and $\left(-\infty, x_{1}\right),\left(x_{N}, \infty\right)$,

and $v$ is negative on $\left(-\infty, x_{1}\right)$ and changes sign on consecutive intervals $\}$.

Of course one could equally well treat the set where $v$ is positive on $\left(-\infty, x_{1}\right)$.

Remark 1.2 ( $N$ is fixed) We only consider the case of a finite number of zeros and our constants are allowed to depend on $N$. Clearly some of our results hold for $N=\infty$ locally in between zeros, but exploring this regime is not the subject of this paper.

Remark $1.3(N=1)$ For the manifold $\mathcal{N}_{1}$ there is no length parameter. Formally, one should think of the "distance between zeros" in this case as $\infty$. We prove the metastability (in this case, stability) result for $N=1$ (and $N=0$ ) separately in Theorem 1.11, below.

Notice for reference below that an energy-optimal profile $v$ satisfies the Euler-Lagrange equation

$$
-v_{x x}+G^{\prime}(v)=0 \quad \text { on } \quad \mathbb{R} \backslash\left(\cup_{i=1}^{N} x_{i}\right)
$$

and is continuous but not $C^{1}$ at the zeros. If we fix $N$ zeros $\mathbf{x}:=\left(x_{1}, \ldots, x_{N}\right)$ with $x_{i+1}-x_{i} \geq$ $\ell$, then the associated energy-optimal profile in $\mathcal{N}_{N}(\ell)$ is uniquely determined. Moreover, we define the "distance of $\mathbf{x}$ " as

$$
\min _{i \in\{1, \ldots, N\}}\left(x_{i+1}-x_{i}\right) \quad(\text { or } \infty \text { in the case } N=0,1) .
$$

The metastable set with which we will work is the set of functions with order one energy and order one $L^{2}$-distance to a member of the slow manifold:

$$
\begin{aligned}
\mathcal{M}_{N}\left(\ell, C_{H}, C_{E}\right):= & \left\{u \in \dot{H}^{1}(\mathbb{R}) \text { such that } E(u) \leq C_{E}\right. \text { and } \\
& \text { there exists } \left.\bar{v} \in \mathcal{N}_{N}(2 \ell) \text { such that }\|u-\bar{v}\|_{L^{2}(\mathbb{R})}^{2} \leq C_{H}\right\} .
\end{aligned}
$$

From now on, all integrals and norms are over $\mathbb{R}$ unless otherwise indicated. 
Remark 1.4 We remark that for $N$ even and $u \in \mathcal{M}_{N}$, there holds

$$
u( \pm \infty)=-1
$$

while for $N$ odd and $u \in \mathcal{M}_{N}$, there holds

$$
u( \pm \infty)= \pm 1 .
$$

Indeed, it is not hard to check that $u-v \in H^{1}$ and then the boundary condition at $\infty$ follows from

$$
\sup _{x \geq M}(f(x))^{2} \leq \int_{M}^{\infty} f^{2}+f_{x}^{2} d x, \quad \text { for } f=u-v,
$$

and analogously for the boundary condition at $-\infty$.

Note that $u \in \mathcal{M}_{N}$ and the associated $\bar{v} \in \mathcal{N}_{N}(2 \ell)$ do not typically have the same zeros. Nonetheless, we observe in the following lemma that $u$ does have $N$ zeros that are separated by at least $\ell$ and that the energy-optimal profile with exactly these zeros is not too far away.

Notation 1.5 We will use the notation $|\boldsymbol{x}-\overline{\boldsymbol{x}}|$ for $\boldsymbol{x}=\left(x_{1}, \ldots, x_{N}\right), \overline{\boldsymbol{x}}=\left(\bar{x}_{1}, \ldots, \bar{x}_{N}\right)$ to denote the maximum distance between the zeros, i.e., by

$$
|\boldsymbol{x}-\overline{\boldsymbol{x}}|:=\max _{i \in 1, \ldots, N}\left|x_{i}-\bar{x}_{i}\right| .
$$

Lemma 1.6 For any $C_{H}, C_{E} \in(0, \infty)$, there exists $\ell_{1} \in(0, \infty)$ with the following property. For any $\ell \geq \ell_{1}$ and $u \in \mathcal{M}_{N}\left(\ell, C_{H}, C_{E}\right)$, let $\overline{\boldsymbol{x}}$ denote the zeros of an associated $\bar{v} \in \mathcal{N}_{N}(2 \ell)$ and $\bar{H}:=\|u-\bar{v}\|_{L^{2}}^{2}$. Then $u$ has $N$ zeros $\boldsymbol{x}=\left\{x_{1}, \ldots, x_{N}\right\}$ such that

$$
|\boldsymbol{x}-\overline{\boldsymbol{x}}| \lesssim \bar{H}+\bar{H}^{\frac{1}{3}} .
$$

Moreover, for the energy-optimal profile $v$ associated to $\boldsymbol{x}$, there holds $v \in \mathcal{N}_{N}(\ell)$ and

$$
\|u-v\|_{L^{2}}^{2} \lesssim \bar{H}+\bar{H}^{\frac{1}{3}} .
$$

We prove Lemma 1.6 in Sect. 3.1. For an explanation of the $\lesssim$ notation, see Notation 1.12, below.

Remark 1.7 Here the existence of $N$ zeros with the given property does not rule out-and is not disturbed by-the existence of additional zeros of $u$. We do not assume that the zeros $\mathbf{x}$ associated by Lemma 1.6 to a solution are uniquely determined or continuous in time, however we will show that after an initial relaxation stage, $u$ has precisely $N$ zeros. As a consequence, $\mathbf{x}$ and $v$ are then uniquely defined and continuous in time until two or more kinks come too close to apply Theorem 1.8. See also Remark 1.10.

Throughout the paper we will for $u \in \mathcal{M}_{N}\left(\ell, C_{H}, C_{E}\right)$ let $\mathbf{x}$ and $v$ denote the zeros and the corresponding energy-optimal profile, respectively, as in Lemma 1.6. In particular, $\mathbf{x}$ and $v$ depend on time. We introduce the $v$-related quantities

$$
\mathcal{E}(t):=E(u)-E(v) \quad \text { and } \quad f(t):=u-v .
$$

In addition the lengthscale $\ell(t)$ of a solution $u$ is defined as the minimum distance between neighboring zeros of $\mathbf{x}$ (defined in Lemma 1.6) or $\infty$ in the case $N=1$. 

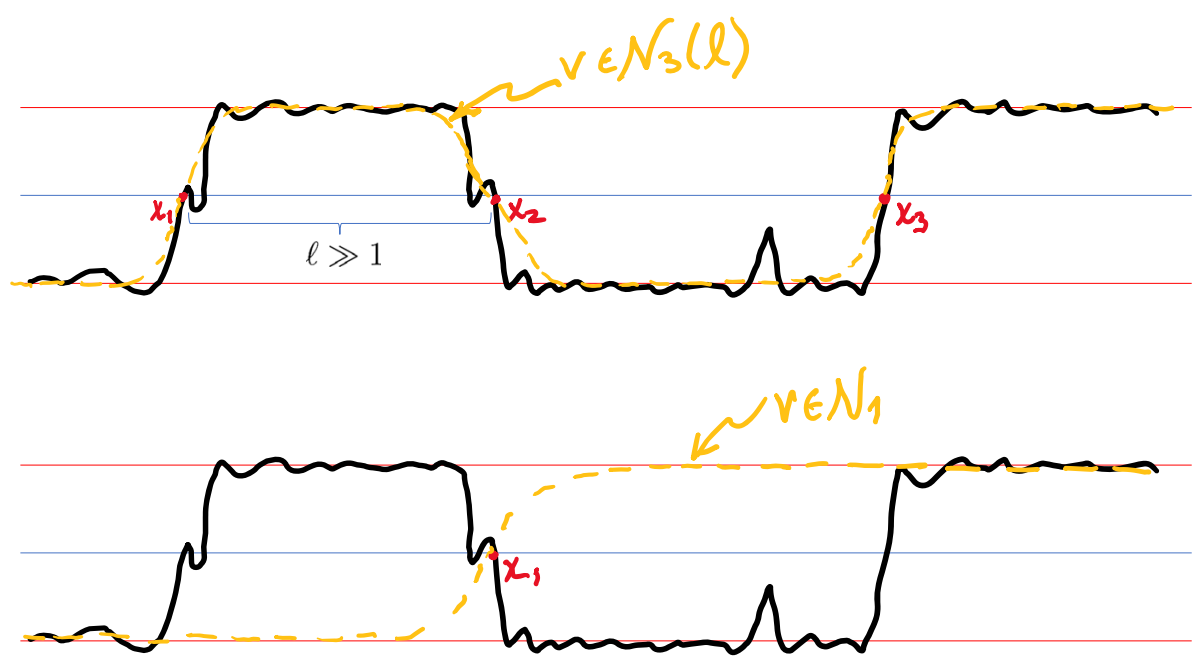

Fig. 1 A rough initial data and two possible choices of slow manifold

For initial data $u_{0} \in \mathcal{M}_{N}$ and the associated (constant in time) function $\bar{v}$, we will for the solution $u$ of the Allen-Cahn equation (1.1) define the $\bar{v}$-related quantities

$$
\overline{\mathcal{E}}(t):=E(u)-E(\bar{v}), \quad \bar{f}(t):=u-\bar{v}, \quad \text { and } \quad \bar{H}(t):=\|u-\bar{v}\|_{L^{2}}^{2} .
$$

We define in addition the dissipation

$$
D(t):=\int\left(u_{x x}-G^{\prime}(u)\right)^{2} d x .
$$

Notice that because of the gradient flow structure and since $\bar{v}$ is fixed in time, there holds

$$
-\frac{d}{d t} \overline{\mathcal{E}}=D
$$

and $\overline{\mathcal{E}}$ is hence decreasing in time, whereas $\mathcal{E}$ is not. Finally, we define the constant

$$
C_{G}:=\sqrt{G^{\prime \prime}( \pm 1)},
$$

which gives the sharp exponential timescale of metastability (see (1.17)).

We sketch the idea of our result before stating the theorems.

We associate to a given initial datum an $N$ and a $v$; in Fig. 1, we sketch a possible $v \in \mathcal{N}_{3}$ and another $v \in \mathcal{N}_{1}$ for a given, rough initial datum. In the upper picture, the energy gap and initial $L^{2}$ distance should appear "not small," and yet much smaller than the distance $\ell$ between the closest zeros. Our theorem will show that the zeros remain for a long time (exponential in $\ell$ ) within an order one neighborhood of their starting positions. The zeros may jump initially; for instance $x_{1}$ or $x_{2}$ may disappear while one of the nearby zeros survives; we do not attempt to track their path and are not bothered by small jumps. The solution quickly relaxes to a state not far from $v$ and evolves slowly after this initial phase. This description is made mathematically precise in Theorem 1.8 below. In the lower sketch, we have chosen $v \in \mathcal{N}_{1}$. Naturally the energy gap and above all the $L^{2}$ distance to this function are very large! Nonetheless, our result proves the following: The solution converges to some $v_{1}(\cdot-c)$ as $t \rightarrow \infty$ and the distance of $c$ to the initial zero is bounded in terms of the initial energy 
gap and $L^{2}$ distance. Because the long-time convergence for $N=0$ and $N=1$ is slightly different from the metastability for $N \geq 2$, we handle the former separately in Theorem 1.11, below.

Theorem 1.8 Let $N \geq 2$ be a natural number and $C_{H}, C_{E} \in(1, \infty)$. Let $C_{\mathrm{ed}}$ be the constant from Lemma 2.1. There exists $\ell_{1} \in(1, \infty), \varepsilon \in(0,1)$ such that for any

$$
\ell_{0} \geq \ell_{1}, \quad \bar{H}_{0} \leq C_{H}, \quad E_{0} \leq C_{E},
$$

the following holds true. The solution $u(t)$ of the Allen-Cahn equation (1.1) with initial condition $u_{0} \in \mathcal{M}_{N}\left(\ell_{0}, \bar{H}_{0}, E_{0}\right)$ satisfies the following. For

$$
\delta=C\left(C_{E}, C_{H}\right) \exp \left(-C_{G} \ell(0)\right) \text { and for all } t \leq \varepsilon \delta^{-1},
$$

the solution is in $\mathcal{M}_{N}\left(\ell_{0}, \bar{H}(t), E_{0}\right)$ and $\bar{H}(t) \lesssim C_{E}, C_{H} \mathcal{E}_{0}+\mathcal{E}_{0}^{\frac{1}{2}}+\bar{H}_{0}$. Moreover the following phases of the evolution exist.

(i) There exists a time $s_{1} \in(0, \infty)$ and a constant $C \in(0, \infty)$ (both depending on $C_{E}$ and $\left.C_{H}\right)$ such that on $\left[0, s_{1}\right]$ there holds

$$
\begin{aligned}
\|u(t)-v(t)\|_{H^{1}}^{2}+\mathcal{E}(t) & \lesssim C_{E}, C_{H} \mathcal{E}_{0} \exp (-t / C)+\delta, \\
|\boldsymbol{x}(t)-\boldsymbol{x}(0)| & \lesssim C_{E}, C_{H} \mathcal{E}_{0}+\mathcal{E}_{0}^{\frac{1}{4}} \\
\|u(t)-u(0)\|_{L^{2}} & \lesssim C_{E}, C_{H} \mathcal{E}_{0}^{\frac{1}{2}}+\mathcal{E}_{0}^{\frac{1}{4}}
\end{aligned}
$$

and such that the energy gap at time $s_{1}$ satisfies

$$
\mathcal{E}\left(s_{1}\right) \leq e_{*} .
$$

Above, as indicated, the constants in $\lesssim$ depend on $C_{H}$ and $C_{E}$. Below all constants are universal.

(ii) There exists an order one time $s_{2}$ (independent of $C_{H}$ and $C_{E}$ ) such that on $\left[s_{1}, s_{1}+s_{2}\right]$, there holds

$$
\begin{aligned}
\|u(t)-v(t)\|_{H^{1}}^{2}+\mathcal{E}(t) & \lesssim \exp \left(-\left(t-s_{1}\right) / C_{e d}\right)+\delta, \\
\left|\boldsymbol{x}(t)-\boldsymbol{x}\left(s_{1}\right)\right| & \lesssim \mathcal{E}\left(s_{1}\right)+\mathcal{E}\left(s_{1}\right)^{\frac{1}{4}}, \\
\left\|u(t)-u\left(s_{1}\right)\right\|_{L^{2}} & \lesssim \delta\left(t-s_{1}\right)\left(\mathcal{E}\left(s_{1}\right)^{\frac{1}{2}}+\mathcal{E}\left(s_{1}\right)^{\frac{1}{4}}\right)
\end{aligned}
$$

and at time $s_{1}+s_{2}$ the solution u satisfies $\mathcal{E}+D \leq \gamma$ for the constant $\gamma$ from Lemma 2.8 and has has exactly $N$ simple zeros, which move continuously in time.

(iii) There exists $s_{3} \sim \ell(0)$ and $\varepsilon>0$ (depending on $C_{E}, C_{H}$ ) such that for $t \in\left[s_{1}+\right.$ $\left.s_{2}, \varepsilon \delta^{-1}\right]$, the solution has exactly $N$ simple zeros and is exponentially close to the slow manifold at $s_{3}$ and slowly evolving in the sense that

$$
\begin{aligned}
\|u(t)-v(t)\|_{H^{1}}^{2}+\mathcal{E}(t) & \lesssim \delta^{2} \quad \text { for } t \geq s_{3}+s_{1}, \\
\left|\boldsymbol{x}(t)-\boldsymbol{x}\left(s_{1}+s_{2}\right)\right| & \lesssim \mathcal{E}\left(s_{1}+s_{2}\right)^{\frac{1}{2}}+\delta\left(t-\left(s_{1}+s_{2}\right)+1\right), \\
\left\|u(t)-u\left(s_{1}+s_{2}\right)\right\|_{L^{2}} & \lesssim \mathcal{E}\left(s_{1}+s_{2}\right)^{\frac{1}{2}}+\delta\left(t-\left(s_{1}+s_{2}\right)+1\right) .
\end{aligned}
$$

Moreover the dissipation is also exponentially small of order $\delta^{2}$ for $t \in\left[s_{1}+s_{3}, \varepsilon \delta^{-1}\right]$. (For notational simplicity we have absorbed the order one constant $s_{2}$ into $s_{3}$.) 
The theorem is proved in Sect. 2.2.

We are particularly interested in placing only weak conditions on the initial data. However in the case of initial data that are "not far" (in the sense of (1.21)) or even close to the slow manifold (as has typically been studied in the literature), more can be said. In particular, a direct application of the metastability framework captures the slowness of the evolution. The parameter $\gamma$ from Lemma 2.8 quantifies what it means for initial data to be well-prepared.

Corollary 1.9 (Small initial data) For initial data that satisfy (1.21) (or, more generally, have initial energy gap strictly less than $2 e_{*}$ ), our result begins directly with stage (ii), so that all constants are universal and we obtain the sharp metastability timescale

$$
T=\varepsilon \exp \left(C_{G} \ell(0)\right)
$$

for an order-one constant $\varepsilon$.

For initial data such that are "well-prepared" in the sense that $\mathcal{E}_{0} \leq \gamma$, the solution enters stage (iii) by time $t=1$. Then the zeros hardly move (cf. (1.26)) and not much happens for a long time in the sense that:

$$
\inf \left\{t>0:\|u(t)-u(0)\|_{L^{2}}=2 \gamma\right\} \gtrsim \delta^{-1} \sim \exp \left(C_{G} \ell(0)\right) .
$$

Proof In the first case, one can set $s_{1}=0$ and begin directly in stage (ii). In the second case, one can set $s_{1}=0$ and Lemma 2.8 and the proof of Theorem 1.8 show that $s_{2} \leq 1$ and the solution enters stage (iii) by time $t=1$. In both cases $\delta \lesssim \exp \left(-C_{G} \ell(0)\right)$.

Remark 1.10 (Going through collisions) Because the solution ends up trapped near an energyoptimal-profile (in energy and $H^{1}$ ), it is forced (by monotonicity of the energy and continuity of the zeros after energy and dissipation have become small) to remain well-approximated by such a profile until two (or more) zeros come close. Our result does not track the detailed dynamics of the kinks (which has been studied elsewhere, for instance in $[10,14]$ on a compact domain and [12] on the line), but it does capture collisions in the following sense.

At time $\varepsilon \delta^{-1}$, the solution is very close to a collection of $N$ kinks. Generically, one pair, say $x_{i}$ and $x_{i+1}$, is closest, and if the separation distance of

$$
\left\{x_{1}, x_{2}, \ldots, x_{i-1}, x_{i+2}, \ldots, x_{N}\right\}
$$

is sufficiently large, one can reapply the theorem at this initial time with the slow manifold $\mathcal{N}_{N-2}$ (and the cycle repeats). See Fig. 2 for an example. The initial data is not far in energy and $L^{2}$ from $\mathcal{N}_{4}(\ell(0))$. If the solution evolves to the state pictured in the lower figure, one can then connect to $\mathcal{N}_{2}$. Notice that the choice of $\hat{x}_{1}$ is not unique; any of the three left-most zeros can be used.

However it may happen that, for a given initial condition, the separation of the zeros at time $\varepsilon \delta^{-1}$ only allows the application of the theorem for $\mathcal{N}_{M}$ with $M \in\{0,1, \ldots, N-4\}$, and in this case we may miss certain intermediate steps.

Nonetheless we show in Theorem 1.11 below that it is always possible to connect to the final state, determined by the boundary condition, of $N=0$ or $N=1$. In particular, we obtain convergence as $t \rightarrow \infty$ to either $v_{\infty} \equiv-1$ or $v_{\infty}=v_{1}(x-c)$ for $c \in \mathbb{R}$.

Theorem 1.11 Let $N=1$ and let $C_{E}, C_{H} \in(1, \infty)$. The solution $u(t)$ of the Allen-Cahn equation (1.1) with initial data $u_{0}$ such that $E_{0} \leq C_{E}$ and $\bar{H}_{0} \leq C_{H}$ satisfies the following. There exists a constant $C \in(0, \infty)$ (depending on $C_{E}$ and $C_{H}$ ) such that 

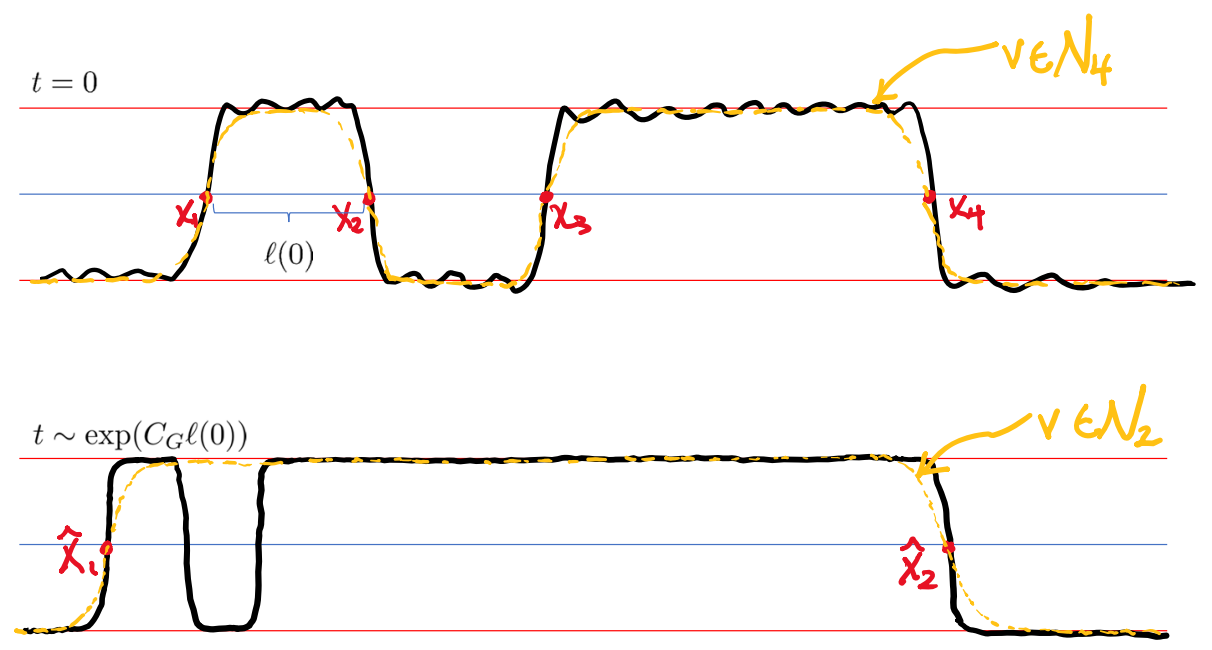

Fig. 2 An example in which Theorem 1.8 would capture the drop from $\mathcal{N}_{4}$ to $\mathcal{N}_{2}$

$$
\begin{aligned}
\|u(t)-v(t)\|_{H^{1}}^{2}+\mathcal{E}(t) & \lesssim_{C_{E}, C_{H}} \mathcal{E}_{0} \exp (-t / C), \\
|\boldsymbol{x}(t)-\boldsymbol{x}(0)| & \lesssim_{C_{E}, C_{H}} \mathcal{E}_{0}+\mathcal{E}_{0}^{\frac{1}{4}}, \\
\|u(t)-u(0)\|_{L^{2}} & \lesssim_{C_{E}, C_{H}} \mathcal{E}_{0}^{\frac{1}{2}} .
\end{aligned}
$$

In particular, there exists $s_{1}<\infty$ (depending on $C_{E}$ and $\left.C_{H}\right)$ such that

$$
\mathcal{E}\left(s_{1}\right) \leq e_{*},
$$

and for $t \geq s_{1}$ there holds

$$
\|u(t)-v(t)\|_{H^{1}}^{2}+\mathcal{E}(t) \lesssim \mathcal{E}\left(s_{1}\right) \exp \left(-\left(t-s_{1}\right) / C_{e d}\right)
$$

for the universal constant $C_{e d}$ from Lemma 2.1. Moreover, there exists $c \in \mathbb{R}$ such that

$$
\boldsymbol{x}(t) \rightarrow c \text { and } u \rightarrow v(\cdot-c) \text { in } H^{1} \quad \text { for } t \rightarrow \infty .
$$

Similarly, for $N=0$ and $C_{E}, C_{H}$ as above, there exists $C \in(0, \infty)$ as above such that

$$
E(u(t)) \lesssim C_{E}, C_{H} E_{0} \exp (-t / C) \text { and }\|u-(-1)\|_{H^{1}} \quad \text { for } t \rightarrow \infty .
$$

We prove the theorem in Sect. 2.3.

Notation 1.12 Throughout the paper we use the notation

$$
A \lesssim B
$$

if there exists a universal constant $C \in(0, \infty)$ such that $A \leq C B$ for $\ell$ large, where $C$ is allowed to depend at most on the potential $G$, the number $N$ of zeros, and the constant $\epsilon$ from Lemma 2.1. In the case of nonuniversal constants, such as dependence on $C_{E}$, we will indicate this by, for example, notation such as $\lesssim C_{E}$ or an explicit remark.

We occasionally use $C$ to represent a constant whose definition may change from line to line and for exponentially small terms when we are not interested in a sharp constant. 


\subsection{Additional Remarks on the Literature}

As remarked above, metastability of the Allen-Cahn equation was already studied by Carr and Pego [10] and Fusco and Hale [14]. In [10], a careful spectral analysis is used to establish, moreover, that initial data starting exponentially close to an appropriately defined slow manifold stays exponentially close for a time that is exponentially large in $\ell$ (the distance between the two closest zeros)—with the optimal constant $C_{G}$ in the exponential.

Bronsard and Kohn [8] introduced a natural and elementary energy method that was able to relax the condition on the initial data, at the cost of a less sharp result on the slow motion. Specifically, they showed that initial data that is algebraically close to the slow manifold stays close to the slow manifold for a time that is algebraically long.

A later analysis by Chen [11] characterizes several stages of the evolution, including the initial relaxation phase and the exponentially slow phase with the sharp constant for the exponential timescale. Chen's analysis uses classical PDE-techniques (including maximum principles). The setting is somewhat different from ours, since for any initial data, the equation with an appropriately small sharp interface parameter is studied. In contrast, our analysis allows for more general data, such as functions with "spurious zeros" that vanish without developing into transition layers.

The metastability framework introduced in [17] is used in that paper, together with a buckling argument, to show that for the Allen-Cahn equation on the torus, for initial data that is order one away from the slow manifold, exponential closeness to the slow manifold is generated and subsequently propagated for an exponentially long time, again with the sharp constant $C_{G}$ in the exponential. This paper revisits that setting on the line and for more general initial conditions.

There is a vast literature on additional aspects of the Allen-Cahn equation, such as the dynamics of collision events, behavior in higher space dimensions or systems, and stochastic perturbations; we do not attempt to summarize those efforts here.

We have focused here on literature on the Allen-Cahn equation, since this is the equation we study below. For references on the Cahn Hilliard equation, we refer to $[1-4,6,7,9,13,15$, $16,18-21]$ and the references therein.

\section{Method and Proof of Main Result}

The first ingredient for the metastability proof are the following nonlinear energy and dissipation estimates.

Lemma 2.1 For any $C_{H}, C_{E} \in(0, \infty)$, there exists $\ell_{1} \in(0, \infty)$ such that, for any $\ell \geq \ell_{1}$, $N \in \mathbb{N}, u \in \mathcal{M}_{N}\left(\ell, C_{H}, C_{E}\right)$, and corresponding $v \in \mathcal{N}_{N}(\ell)$ as in Lemma 1.6, there holds:

$$
\begin{aligned}
& \|u-v\|_{H^{1}}^{2} \lesssim C_{H}, C_{E} \mathcal{E} \lesssim C_{E}\|u-v\|_{H^{1}}^{2}, \\
& \|u-v\|_{H^{1}}^{2} \lesssim C_{H_{H}, C_{E}} D .
\end{aligned}
$$

Moreover, for any $\epsilon>0$ there exists $\ell_{1} \in(0, \infty)$ such that for $\ell$ and $u$ as above, an energy gap $\mathcal{E}(u) \leq 2 e_{*}-\epsilon$ implies that (2.1) and (2.2) hold with a constant $C_{e d}$ that is independent of $C_{H}$ and $C_{E}$.

The proofs of the energy and dissipation estimates are long but elementary. The proof of the energy gap estimates can be found (with no assumption that the zeros are simple or that $u$ is of one sign between zeros) via a slight modification (using Lemma 1.6 above) of 
[20, Proof of Lemma 2.1, Equation (2.1)], and we do not repeat the proof here. We include the improvement to universal constants for energy gap below $2 e_{*}-\epsilon$ and the proof of the dissipation estimate in the Appendix.

The second ingredient for the metastability proof is a Lipschitz condition on $\mathcal{N}_{N}(\ell)$. To transfer information back and forth between shifts in zeros and shifts in $L^{2}$, we need the following elementary lemma, a proof of which can be found in [17].

Lemma 2.2 (Lemma 3.2 of [17]) There exists $\ell_{1}, C_{1} \in(0, \infty)$ such that $\ell \geq \ell_{1}$ and for any two $v, \tilde{v} \in \mathcal{N}_{N}(\ell)$ with zeros $\boldsymbol{x}, \tilde{\boldsymbol{x}}$, the following holds true. If $|\boldsymbol{x}-\tilde{\boldsymbol{x}}| \leq \ell / C_{1}$, then

$$
|\boldsymbol{x}-\tilde{\boldsymbol{x}}| \lesssim\|v-\tilde{v}\|_{L^{2}}^{2}+\|v-\tilde{v}\|_{L^{2}} \text { and }\|v-\tilde{v}\|_{L^{2}} \lesssim|\boldsymbol{x}-\tilde{\boldsymbol{x}}|+1 .
$$

Using this lemma, the Lipschitz condition can be formulated as follows. The Lipschitz condition was used already in [10] and a self-contained proof can also be found in [17, Lemma 3.1].

Lemma 2.3 There is $C, \ell_{1} \in(0, \infty)$ with the following property. For any $\ell \geq \ell_{1}, N \in \mathbb{N}$, and $v, \tilde{v} \in \mathcal{N}_{N}(\ell)$, let $\boldsymbol{x}$ and $\tilde{\boldsymbol{x}}$ denote the zeros of $v$, $\tilde{v}$. There holds

$$
|E(v)-E(\tilde{v})| \lesssim \delta|\boldsymbol{x}-\tilde{\boldsymbol{x}}| \text { with } \delta:=\exp \left(-C_{G} \ell\right) .
$$

In particular, there exists $C_{1} \in(0, \infty)$ with the following property. For every $C_{2} \in(0, \infty)$, there exists $C_{3} \in(0, \infty)$ such that for $v, \tilde{v} \in \mathcal{N}_{N}(\ell)$ with $|\boldsymbol{x}-\tilde{\boldsymbol{x}}| \leq \ell / C_{1}$ and $\|v-\tilde{v}\|_{L^{2}} \leq C_{2}$, there holds

$$
|\boldsymbol{x}-\tilde{\boldsymbol{x}}| \leq C_{3}\|v-\tilde{v}\|_{L^{2}} \text { and }|E(v)-E(\tilde{v})| \leq \delta C_{3}\|v-\tilde{v}\|_{L^{2}} .
$$

We now state (without proof) two metastability propositions; below we refer specifically to the proofs from [20]. The second proposition-Proposition 2.5-is a slight generalization of the original result from [17, Theorem 1.1]. On a time interval $\left[s_{1}+1, T\right]$ during which the solution $u$ has $N$ simple zeros, we use this result to deduce that the solution enters a $\delta^{2}$ neighborhood of the slow manifold.

The simpler Proposition 2.4 captures metastability by establishing exponential in time convergence of $\overline{\mathcal{E}}$ (instead of $\mathcal{E}$ ). In so doing, it delivers the sharp factor of 2 in (2.9) - at the expense of an error term that goes like $\delta$ (instead of $\delta^{2}$ ); compare to (2.14). We use this result initially in time.

We will apply the propositions to the Allen-Cahn equation with $\mathcal{N}=\mathcal{N}_{N}\left(\ell_{0}\right)$ and $\|\cdot\|_{0}=$ $\|\cdot\|_{1}=\|\cdot\|_{L^{2}}$.

Proposition 2.4 (Proposition 2.9 of [20]) Let $\left(X_{0},\|\cdot\|_{0}\right)$ be a Banach space and $\left(X_{1},\|\cdot\|_{1}\right)$ be a Hilbert space with

$$
X_{1} \subset X_{0} \quad \text { and } \quad\|u\|_{0} \leq\|u\|_{1} .
$$

For a differentiable functional $E: X_{1} \rightarrow \mathbb{R}$, consider the gradient flow

$$
\dot{u}=-\nabla E(u) \quad u(0)=u_{0} .
$$

Let $\mathcal{M} \subset X_{1}$ and suppose that there is a set $\mathcal{N} \subset X_{0}$ with the following properties:

(i) For every $u \in \mathcal{M}$ there is a $v \in \mathcal{N}$ such that

$$
\frac{1}{2}\|u-v\|_{0}^{2} \leq E(u)-E(v) \leq \frac{1}{2}\|\nabla E(u)\|_{1}^{2} .
$$


(ii) There is a constant $\delta \in(0,1)$ such that for all $v_{1}, v_{2} \in \mathcal{N}$ we have

$$
\left|E\left(v_{1}\right)-E\left(v_{2}\right)\right| \leq \delta\left\|v_{1}-v_{2}\right\|_{0} .
$$

Suppose that for $t \in[0, T]$, the solution of (2.6) satisfies $u(t) \in \mathcal{M}$ and let $v(t) \in \mathcal{N}$ denote a function associated to the solution at time $t$ as in (i). Define the initial quantity $e_{0}:=E(u(0))-E(v(0))$ and suppose that $\delta$ is small enough so that $\ln \left(e_{0}^{\frac{1}{2}} / \delta\right) \leq \delta^{-1}$. Then for all $t \leq \delta^{-1}$ there holds

$$
|E(u(t))-E(v(0))| \lesssim \exp (-2 t) e_{0}+\delta\left(e_{0}^{\frac{1}{2}}+e_{0}^{\frac{1}{4}}\right) .
$$

Furthermore, changes up to time $T_{1}:=\max \left\{0,2 \ln \left(\frac{e_{0}}{\delta}\right)\right\}$ are controlled by

$$
\sup _{t \leq T_{1}}\left(\|u(t)-u(0)\|_{1}+\|v(t)-v(0)\|_{0}\right) \lesssim e_{0}^{\frac{1}{2}}+e_{0}^{\frac{1}{4}},
$$

and the energy gap at $t=T_{1}$ is small:

$$
\left|E\left(u\left(T_{1}\right)\right)-E(v(0))\right|+\left|E\left(u\left(T_{1}\right)\right)-E\left(v\left(T_{1}\right)\right)\right| \lesssim \delta\left(e_{0}^{\frac{1}{2}}+e_{0}^{\frac{1}{4}}\right) .
$$

For times $T_{1} \leq t \leq \delta^{-1}$ the motion is slow in the sense that

$$
\begin{aligned}
& \left\|u(t)-u\left(T_{1}\right)\right\|_{1}^{2} \lesssim \delta\left(t-T_{1}\right)\left(e_{0}^{\frac{1}{2}}+e_{0}^{\frac{1}{4}}\right), \\
& \left\|v(t)-v\left(T_{1}\right)\right\|_{0}^{2} \lesssim \delta\left(1+t-T_{1}\right)\left(e_{0}^{\frac{1}{2}}+e_{0}^{\frac{1}{4}}\right)+\delta^{2},
\end{aligned}
$$

and the solution is trapped near the slow manifold via

$$
\|u(t)-v(t)\|_{0}^{2}+|E(u(t))-E(v(t))| \lesssim \delta\left(e_{0}^{\frac{1}{2}}+e_{0}^{\frac{1}{4}}+\left\|v(t)-v\left(T_{1}\right)\right\|_{0}\right) .
$$

The second result is a slight generalization of [17, Theorem 1.1], which appeared already in [20], although we state the result in more detail, because of its interest for our current application.

Proposition 2.5 (Proposition 2.10 of [20]) Let $X_{0}, X_{1}, E, \mathcal{M}, \mathcal{N}, u, v, T, e_{0}$, and $\delta$ be as in Proposition 2.4. Suppose moreover that $t \mapsto E(v(t))$ is integrable on $[0, T]$. Then for every $\epsilon \in(0,1)$ there is a constant $C_{\epsilon}$ such that

$$
\begin{gathered}
\|u(t)-v(t)\|_{0}^{2}+E(u(t))-E(v(t)) \\
\lesssim \exp (-(2-\epsilon) t) e_{0}+C_{\epsilon} \delta^{2} .
\end{gathered}
$$

Furthermore, let $T_{1}:=\max \left\{0, \ln \left(\mathcal{E}_{0} / \delta^{2}\right)\right\}$. For $0 \leq t \leq T_{1}$, there holds

$$
\sup _{t \leq T_{1}}\left(\|u(t)-u(0)\|_{1}+\|v(t)-v(0)\|_{0}\right) \lesssim e_{0}^{\frac{1}{2}},
$$

and for $T_{1}<t \leq T$ there holds

$$
\left\|u(t)-u\left(T_{1}\right)\right\|_{1}+\left\|v(t)-v\left(T_{1}\right)\right\|_{0} \lesssim \delta\left(t-T_{1}+1\right)
$$




\subsection{Postprocessing: Small Dissipation and Simple Zeros}

In order to deduce that the solution has simple zeros after the energy has relaxed, we derive the following differential inequality for the dissipation.

Lemma 2.6 For any $C_{H}, C_{E} \in(0, \infty)$, there exist $\varepsilon, \ell_{1} \in(0, \infty)$ such that, for any $\ell \geq \ell_{1}$, $N \in \mathbb{N}$, and a solution $u \in \mathcal{M}_{N}\left(\ell, C_{H}, C_{E}\right)$ of the Allen-Cahn equation (1.1), the following holds true. Suppose that for $v \in \mathcal{N}_{N}(2 \ell)$ as in Lemma 1.6, there holds $\|u-v\|_{\infty} \leq \varepsilon$. Then

$$
\frac{\mathrm{d}}{\mathrm{d} t} D \lesssim D
$$

We give the proof in Sect. 3.2 below. Smallness of the dissipation based on smallness of the energy gap is deduced as in [20, Lemma 2.12].

Lemma 2.7 Let $C_{H}, C_{E} \in(0, \infty)$. There exist $\ell_{1}, \gamma_{1} \in \mathbb{R}^{+}$such that if $\ell \geq \ell_{1}$ and $u \in$ $\mathcal{M}_{N}\left(\ell, C_{H}, C_{E}\right)$ satisfies $\overline{\mathcal{E}} \leq \gamma$ on $[s, t]$ for $\gamma \leq \gamma_{1}$ and some $t \geq s+1$, then

$$
\max _{[s+1, t]} D \lesssim \gamma
$$

The proof is identical to the proof of Lemma 2.12 in [20] but for completeness, we include it in Sect. 3.2. As in [20], smallness of the the dissipation then implies simple zeros.

Lemma 2.8 Let $C_{H}, C_{E} \in(0, \infty)$. There exist $\ell_{1}, \gamma \in \mathbb{R}^{+}$such that if $\ell \geq \ell_{1}$ and $u \in$ $\mathcal{M}_{N}\left(\ell, C_{H}, C_{E}\right)$ satisfies $\mathcal{E}+D \leq \gamma$, then u has exactly $N$ simple zeros.

Lemma 2.8 is also proved in Sect. 3.2.

\subsection{Proof of Theorem 1.8 and Corollary 1.9}

We are now ready to prove our main theorem.

In the proof we will choose the lengthscale $\ell_{0}$ between zeros of $\bar{v}$ sufficiently large with respect to $C_{H}, C_{E}$ so that the lengthscale $\ell(0)$ between the zeros of $v(0)$ is of the same order, i.e., $\ell(0) \sim \ell_{0}$; see (2.21) below.

Proof of Theorem 1.8 Step 0 In this preliminary step we collect the facts that we will need about the motion of and distance between zeros and summarize the idea of the proof.

We choose $\ell_{0} \geq \ell_{1}$ for the constant $\ell_{1}$ from the auxiliary lemmas above. To the initial data $u_{0}$ we associate $\bar{v} \in \mathcal{N}_{N}(2 \ell)$, which is fixed throughout the proof. As usual, the zeros of $\bar{v}$ are denoted by $\overline{\mathbf{x}}$. We also choose $\ell_{0}$ large enough so that Lemma 1.6 gives control on the zeros $\mathbf{x}(\cdot)$ of $u$ initially in the form

$$
|\mathbf{x}(0)-\overline{\mathbf{x}}| \leq \frac{\ell_{0}}{4}, \quad \ell(0) \geq \ell_{0},
$$

where $\ell(t)$ as usual denotes the distance between the closest consecutive zeros in $\mathbf{x}(t)$.

The proof will rely on a buckling argument. A priori, we will need to control changes in $\bar{H}(t)$ and $\ell(t)$. To this end, we define the maximal displacement of the zeros as

$$
\Delta \mathbf{x}(t):=\sup _{0 \leq t^{\prime} \leq t}\left|\mathbf{x}\left(t^{\prime}\right)-\mathbf{x}(0)\right|,
$$


and we introduce the constant $C_{3} \in(1, \infty)$, whose dependence on universal constants and $\bar{H}_{0}, E_{0}$ will be clarified below. We define the time:

$$
T_{1}:=\inf \left\{t \geq 0: \bar{H}(t) \geq C_{3}\right\} .
$$

Note that according to (2.19) and $E(u(t)) \leq E_{0}$, the function $u(t) \in \mathcal{M}_{N}\left(\ell_{0}, C_{3}, E_{0}\right)$ and hence, by Lemma 1.6, $v(t)$ is well-defined for all $t \in\left[0, T_{1}\right]$. Note also that according to (1.12) the motion of the zeros on $\left[0, T_{1}\right]$ is bounded according to

$$
\Delta \mathbf{x}(t) \leq C_{4},
$$

where $C_{4}=C_{4}\left(C_{3}\right)$ comes from estimating

$$
|\Delta \mathbf{x}(t)| \leq \sup _{0 \leq t^{\prime} \leq t}\left(\left|\mathbf{x}\left(t^{\prime}\right)-\overline{\mathbf{x}}\right|+|\overline{\mathbf{x}}-\mathbf{x}(0)|\right) \lesssim \bar{H}(t)^{\frac{1}{3}}+\bar{H}(t)+\bar{H}_{0}^{\frac{1}{3}}+\bar{H}_{0} .
$$

According to (2.18) and choosing $\ell_{0}$ sufficiently large with respect to $C_{4}$, we observe that for $t \leq T_{1}$ there holds

$$
\ell_{0} \sim \ell(0) \sim \ell(t)
$$

and $v(t) \in \mathcal{N}_{N}\left(\frac{\ell_{0}}{2}\right)$. At the same time, choosing $\ell_{0}$ sufficiently large with respect to $C_{4}$ allows us to apply Lemma 2.3.

We now control three phases of the evolution. Without loss of generality, we may assume that $\mathcal{E}_{0}>e_{*}$. In the initial phase, we will define $T_{2}:=\inf \left\{t \geq 0: \mathcal{E}(t) \leq e_{*}\right\}$ and $T_{*}:=$ $\min \left\{T_{1}, T_{2}\right\}$. In Step 1 we will obtain

$$
T_{*}<T_{1}, \quad \mathcal{E}\left(T_{*}\right) \leq e_{*} .
$$

The timescale $s_{1}$ in the statement of the theorem is then defined as

$$
s_{1}:=T_{*} .
$$

At this point, the energy gap is "small" and the EED inequalities from Lemma 2.1 hold with the universal constant $C_{e d}$. We will use the solution at $s_{1}$ as initial data for Proposition 2.4 in Step 2 to see that both the energy gap and dissipation can be made small by time $s_{1}+s_{2}$. At this point the solution has $N$ simple zeros that move continuously in time, and we will use Proposition 2.5) to establish and make use of the exponential decay in time of the energy gap in Step 3 on the interval $\left[s_{1}+s_{2}, T_{* *}\right]$ for

$$
T_{* *}:=\min \left\{T_{1}, \varepsilon \exp \left(C_{G} \ell(0)\right)\right\}
$$

for $\varepsilon \in(0,1)$ to be specified below. We will obtain in Step 3 that

$$
T_{* *}<T_{1} \text { and hence } T_{* *}=\varepsilon \exp \left(C_{G} \ell(0)\right) .
$$

Step 1 According to (2.19), (2.20), and the definition of $T_{*}, \Delta \mathbf{x}\left(T_{*}\right)$ is bounded in terms of $C_{4}$. We will show that it is in fact bounded only in terms of $C_{E}, C_{H}$ and that

$$
\begin{aligned}
\mathcal{E}(t) & \leq 2 \mathcal{E}_{0}, \\
\mathcal{E}(t) & \lesssim \exp (-t / C) \mathcal{E}_{0}+\delta\left(\mathcal{E}_{0}^{\frac{1}{2}}+\mathcal{E}_{0}^{\frac{1}{4}}\right), \\
\Delta \mathbf{x}\left(T_{*}\right) & \lesssim \mathcal{E}_{0}+\mathcal{E}_{0}^{\frac{1}{4}}, \\
\|u(t)-u(0)\|_{L^{2}} & \lesssim \mathcal{E}_{0}^{\frac{1}{2}}+\mathcal{E}_{0}^{\frac{1}{4}},
\end{aligned}
$$


for

$$
\delta=\exp \left(-C_{G} \ell(0)\right),
$$

where here and for the rest of Step 1 (only), $\lesssim$ allows for a dependence on $C_{E}$ and $C_{H}$. These estimates (and (2.1)), yield (1.18)-(1.20) for $t \leq T_{*}$.

The first bound is immediate. Indeed, let

$$
\hat{\mathcal{E}}:=E(u)-E(v(0)) .
$$

From $\mathcal{E}_{0} \gtrsim 1$ and

$$
|\mathcal{E}-\hat{\mathcal{E}}| \lesssim \exp (-C \ell)
$$

we obtain

$$
\mathcal{E} \sim \hat{\mathcal{E}}, \quad \hat{\mathcal{E}} \gtrsim 1
$$

Hence monotonic decay of $\hat{\mathcal{E}}$ and (2.29), (2.30) yield (2.25) for $\ell_{1}$ sufficiently large.

We now turn to (2.26)-(2.28). The idea is to apply Proposition 2.4 with $\|\cdot\|_{0}=\|\cdot\|_{1}=$ $\|\cdot\|_{L^{2}}$; hence, we need to verify (2.7) and (2.8). For this, we invoke the EED estimate from Lemma 2.1 in the form:

$$
\frac{1}{C\left(C_{H}, C_{E}\right)}\|u-v\|_{L^{2}}^{2} \leq \mathcal{E} \leq C\left(C_{H}, C_{E}\right) D=C\left(C_{H}, C_{E}\right)\|\nabla E\|_{L^{2}}^{2}
$$

and the Lipschitz condition from Lemma 2.3 in the form:

$$
\begin{aligned}
|E(v)-E(\tilde{v})| & \stackrel{(2.4)}{\lesssim} \tilde{\delta}|\mathbf{x}-\tilde{\mathbf{x}}| \quad \text { with } \quad \tilde{\delta}:=\exp \left(-C_{G} \min \ell(t)\right) \\
& \stackrel{(2.3)}{\lesssim} \tilde{\delta}\left(1+C \Delta \mathbf{x}\left(T_{*}\right)\right)\|v-\tilde{v}\|_{L^{2}} .
\end{aligned}
$$

Here and in the following steps we estimate the minimal interval length via

$$
\min _{t} \ell(t) \geq \ell(0)-\Delta \mathbf{x}\left(T_{*}\right) .
$$

From this estimate we conclude

$$
|E(v)-E(\tilde{v})| \lesssim \delta\|v-\tilde{v}\|_{L^{2}},
$$

for

$$
\delta \lesssim \exp \left(-C_{G} \ell(0)\right)\left(1+C \Delta \mathbf{x}\left(T_{*}\right)\right) \exp \left(C \Delta \mathbf{x}\left(T_{*}\right)\right)
$$

We will, by controlling $\Delta \mathbf{x}\left(T_{*}\right)$, show good control of $\delta$. For a rough initial bound, we estimate

$$
\delta \lesssim C\left(C_{4}\right) \exp \left(-C_{G} \ell(0)\right)
$$

We now rescale time and energy by the constant appearing in (2.31) according to $\tilde{t}=$ $t / C\left(C_{H}, C_{E}\right), \tilde{E}=C\left(C_{H}, C_{E}\right) E$ to obtain a normalized EED inequality as in (2.7) and note that $\delta$ retains the form (2.35), up to replacing $C\left(C_{4}\right)$ by $C\left(C_{4}, C_{H}, C_{E}\right)$.

We now apply Proposition 2.4 in the new variables and then rescale the results back to the original variables. (Note that this induces a dependence of our estimates below-as well as 
in the constant $C$ in (2.26) and the symbol $\lesssim$ in (2.26)-(2.28) - on $C_{H}$ and $C_{E}$.) We obtain the exponential decay of $E(u(t))-E(v(0))$ and the bound

$$
\begin{aligned}
& \sup _{t}\|u(t)-u(0)\|_{L^{2}}+\sup _{t}\|v(t)-v(0)\|_{L^{2}} \\
& \stackrel{(2.10)}{\lesssim}\left(E\left(u_{0}\right)-E(v(0))\right)^{\frac{1}{2}}+\left(E\left(u_{0}\right)-E(v(0))\right)^{\frac{1}{4}}
\end{aligned}
$$

for times up to

$$
\hat{T}_{*}:=\min \left\{T_{*}, \ln \left(\left(E\left(u_{0}\right)-E(v(0))\right) / \delta\right)\right\} .
$$

The triangle inequality and (2.36) allow us to conclude that, for $C_{3}$ large enough with respect to $C_{H}$ and $C_{E}$, there holds $T_{1}>\hat{T}_{*}$. In addition, plugging (2.36) into (2.3) implies that

$$
\Delta \mathbf{x}\left(\hat{T}_{*}\right) \lesssim \hat{\mathcal{E}}(0)+\hat{\mathcal{E}}(0)^{\frac{1}{4}} \stackrel{(2.30)}{\lesssim} \mathcal{E}(0)+\mathcal{E}(0)^{\frac{1}{4}}
$$

which improves our estimate of $\delta$, so that (2.34) holds with

$$
\delta \lesssim C\left(C_{H}, C_{E}\right) \exp \left(-C_{G} \ell(0)\right) .
$$

On the one hand, using the exponential in time decay of the energy gap (cf. (2.9), (2.30)) and for $\ell_{0}$ sufficiently large, we obtain

$$
\hat{T}_{*}=\min \left\{T_{*}, \ln \left(\left(E\left(u_{0}\right)-E(v(0))\right) / \delta\right)\right\}=T_{*}<\infty,
$$

so that (2.26) holds and $\mathcal{E}\left(T_{*}\right)=e_{*}$. On the other hand (2.37) and (2.36) for times up to $T_{*}$ give (2.27) and (2.28).

Step 2 Once we have reached the energy gap of $e_{*}$ at time $s_{1}$, the EED estimates hold with the universal constant $C_{e d}$ and we begin to examine the relaxation of the energy gap more carefully, taking the solution at time $s_{1}$ as initial data. We consider the evolution on $\left[s_{1}, T_{*}\right]$, where now

$$
\begin{aligned}
& T_{1}:=\inf \left\{t>s_{1}:\left\|u(t)-v\left(s_{1}\right)\right\|_{L^{2}}^{2} \geq C_{3}\right\}, \\
& T_{2}:=\inf \left\{t>s_{1}: \mathcal{E}(t)+D(t) \leq \gamma\right\},
\end{aligned}
$$

for the parameter $\gamma>0$ from Lemma 2.8. Let

$$
T_{*}:=\min \left\{T_{1}, T_{2}\right\} .
$$

Arguing with Proposition 2.4 and a buckling argument as in Step 1, we deduce (1.22)-(1.24) and $T_{*}<T_{1}$. From (1.22) and Lemmas 2.7, 2.8, and choosing $\ell_{0}$ large enough so that $\delta$ is sufficiently small (in relation to $\gamma$ ), we obtain $T_{2}=T_{*}<\infty$ and hence that there exists an order one time $s_{2}$ (proportional to $C_{e d} \ln (\gamma)$ ) such that for $t \geq s_{1}+s_{2}$, the solution $u$ has $N$ simple zeros that move continuously in time. Therefore $E(v(t))$ is measurable and, in particular, integrable, and we may apply Proposition 2.5.

We could use Proposition 2.4 longer and, in particular, until the energy gap has become order $\delta$. However to get the better $\delta^{2}$ closeness to the slow manifold (which occurs on the same $\sim \ell(0)$ timescale), we switch to Proposition 2.5.

Step 3 The buckling argument is again very close to that carried out in Step 1, however this time we will use Proposition 2.5, taking the solution at time $s_{1}+s_{2}$ as initial data. We consider shifts in the zeros after time $s_{1}+s_{2}$ :

$$
\Delta_{S} \mathbf{x}(t):=\sup _{s_{1}+s_{2} \leq t^{\prime} \leq t}\left|\mathbf{x}\left(t^{\prime}\right)-\mathbf{x}\left(s_{1}+s_{2}\right)\right|
$$


and define $T_{1}$ as

$$
T_{1}:=\inf \left\{t>s_{1}+s_{2}:\left\|u(t)-v\left(s_{1}+s_{2}\right)\right\|_{L^{2}}^{2} \geq C_{3}\right\}
$$

and $T_{* *}$ as in (2.23) for $\varepsilon$ to be specified below. We estimate the remaining interval length as in (2.33) via

$$
\begin{array}{r}
\ell(t) \geq \ell(0)-\sup _{t^{\prime} \leq t}\left|\mathbf{x}\left(t^{\prime}\right)-\mathbf{x}(0)\right| \geq \ell(0)-\Delta_{S} \mathbf{x}\left(T_{* *}\right)-\sup _{t^{\prime} \leq s_{1}+s_{2}}\left|\mathbf{x}\left(t^{\prime}\right)-\mathbf{x}(0)\right| \\
\stackrel{(1.19),(1.23)}{\geq} \ell(0)-\Delta_{S} \mathbf{x}\left(T_{* *}\right)-C\left(C_{E}, C_{H}\right),
\end{array}
$$

so that we can apply Proposition 2.5 on $\left[s_{1}+s_{2}, T_{* *}\right]$ with a $\delta$ satisfying

$$
\delta \lesssim C\left(C_{E}, C_{H}\right) \exp \left(-C_{G} \ell(0)\right) \exp \left(C \Delta_{S} \mathbf{x}\left(T_{* *}\right)\right)\left(1+\Delta_{S} \mathbf{x}\left(T_{* *}\right)\right) .
$$

Using

$$
\Delta_{S} \mathbf{x}\left(T_{* *}\right) \stackrel{(2.3)}{\lesssim} \sup _{t \in\left[s_{1}+s_{2}, T_{* *}\right]}\left(\left\|v(t)-v\left(s_{1}+s_{2}\right)\right\|_{L^{2}}+\left\|v(t)-v\left(s_{1}+s_{2}\right)\right\|_{L^{2}}^{2}\right)
$$

and bounding the $L^{2}$ norm via (2.15) and (2.16), we obtain

$$
\begin{aligned}
\Delta_{S} \mathbf{x}\left(T_{* *}\right) & \lesssim \mathcal{E}\left(s_{1}+s_{2}\right)^{\frac{1}{2}}+\delta\left(T_{* *}-\left(s_{1}+s_{2}\right)+1\right) \\
& \lesssim \mathcal{E}\left(s_{1}+s_{2}\right)^{\frac{1}{2}}+\varepsilon C\left(C_{E}, C_{H}\right) \exp \left(C \Delta_{S} \mathbf{x}\left(T_{* *}\right)\right)\left(1+\Delta_{S} \mathbf{x}\left(T_{* *}\right)\right) \\
& \left.\lesssim \mathcal{E}\left(s_{1}+s_{2}\right)^{\frac{1}{2}}+\varepsilon C\left(C_{E}, C_{H}\right) \exp \left(C \Delta_{S} \mathbf{x}\left(T_{* *}\right)\right) \quad \text { (up to a renaming of } C\right) .
\end{aligned}
$$

Continuity of $\Delta_{S} \mathbf{x}$ and choosing $\varepsilon \in(0,1)$ small enough (with respect to $C_{E}, C_{H}$ ) implies

$$
\Delta_{S} \mathbf{x}\left(T_{* *}\right) \lesssim \mathcal{E}\left(s_{1}+s_{2}\right)^{\frac{1}{2}}
$$

Consequently there holds

$$
\delta \lesssim C\left(C_{E}, C_{H}\right) \exp \left(-C_{G} \ell(0)\right)
$$

The remaining bounds follow from (2.14)-(2.16) and Lemmas 2.2 and 2.7.

\subsection{Long-Time Convergence Proof}

The proof of Theorem 1.11 is similar to and simpler than that of Theorem 1.8, so we give a slightly abridged proof. Parts of the proof involve EED arguments (which need to be adjusted to this simpler setting), so the reader unfamiliar with such arguments may wish to read the EED proof from the Appendix before continuing.

Proof of Theorem 1.11 We start with $N=0$. Since the attracting state $v_{\infty} \equiv-1$ is constant and unique (because of the boundary conditions; cf. Remark 1.4) and $E\left(v_{\infty}\right)=0$, there are numerous simplifications. Once we have established EED, we can directly argue

$$
-\frac{d}{d t} E(u)=-\frac{d}{d t} \mathcal{E}(u)=D \lesssim \mathcal{E}(u)=E(u)
$$

to deduce exponential convergence of the energy gap and hence also of $u$ to $v_{\infty}$ in $H^{1}$, where $\lesssim$ incorporates $C_{E}, C_{H}$ dependence or not, depending on the initial condition. 
Hence it suffices to check EED. The upper bound on the energy is easy, since

$$
E(u)=\int\left[\left(u-v_{\infty}\right)_{x}\right]^{2}+G(u) d x \lesssim C_{E}\left\|u-v_{\infty}\right\|_{H^{1}}^{2},
$$

where we have as usual used the $L^{\infty}$ bound on $u$ induced by the energy bound and the trick of Modica and Mortola. To check the lower bound on the energy, we as usual argue that when $E(u) \geq \gamma$, there holds

$$
\left\|u-v_{\infty}\right\|_{L^{2}} \leq C_{H} \leq \frac{C_{H}}{\gamma} E(u) .
$$

On the other hand, for $\gamma \leq 2 e_{*}-\epsilon$, we argue as in the demonstration of universal constants for the energy gap in the proof of Lemma 2.1.

The linearized dissipation estimate is also simplified, since $\mathcal{L}_{A C}=f_{x x}-G^{\prime \prime}\left(v_{\infty}\right) f=$ $f_{x x}-G^{\prime \prime}(-1) f$, and hence

$$
\left\|u-v_{\infty}\right\|_{H^{1}}^{2} \leq \frac{1}{\min \left\{2 G^{\prime \prime}(-1),\left(G^{\prime \prime}(-1)\right)^{2}\right\}} \int\left(\mathcal{L}_{A C}(f)\right)^{2} d x
$$

follows via integration by parts. The arguments for large dissipation and for $L^{\infty}$ closeness in the case of small dissipation carry through just as in the proof of Lemma 2.1.

We turn now to the case $N=1$. Here the slow manifold consists of all shifts of $v_{1}$, and the function $v$ identified in Lemma 1.6 is nothing but the kink $v_{1}$ shifted to share a zero with the solution $u: v=v_{1}(\cdot-\mathbf{x}(t))$. Hence, as for $N=0$, the energy of $v$ is constant in time. EED is already contained within Lemma 2.1, and we deduce immediately (as above for $N=0$ ) that

$$
\|u-v\|_{H^{1}}^{2}+\mathcal{E}(t) \lesssim \mathcal{E}_{0} \exp (-t / C),
$$

where $\lesssim, C$ depend on $C_{E}, C_{H}$ for general initial data and are universal once the energy has become small. It remains only to check that $\mathbf{x}(t)$ converges, but this is also not hard. Once $u$ is well-approximated by a kink-for instance for $t \geq s_{1}$-we can estimate

$$
\begin{aligned}
|\mathbf{x}(t)-\mathbf{x}(t+1)|^{2} & \lesssim\|u(t)-u(t+1)\|_{L^{2}}^{2} \leq\left(\int_{t}^{t+1}|\dot{\mathcal{E}}|^{\frac{1}{2}} d s\right)^{2} \leq \mathcal{E}(t)-\mathcal{E}(t+1) \\
& \stackrel{(2.39)}{\lesssim} \mathcal{E}_{0}(\exp (-t / C)-\exp (-(t+1) / C))=\mathcal{E}_{0} \exp (-t / C)\left(1-e^{-1 / C}\right) .
\end{aligned}
$$

Iterating the above and using the triangle inequality in the form

$$
|\mathbf{x}(t)-\mathbf{x}(s)| \leq|\mathbf{x}(t)-\mathbf{x}(t+1)|+|\mathbf{x}(t+1)-\mathbf{x}(t+2)|+\ldots|\mathbf{x}(t+J)-\mathbf{x}(s)|
$$

for $s \in[t+J, t+J+1]$, for some $J \in \mathbb{N}$, we deduce that $\mathbf{x}(t) \rightarrow c$ for some $c \in \mathbb{R}$ as $t \rightarrow \infty$. From here, using (2.39), we conclude $H^{1}$ convergence of $v$ and $u$ to $v_{1}(\cdot-c)$.

Similarly, we have on the one hand

$$
\|u(t)-u(0)\|_{L^{2}}^{2} \leq\left(\int_{0}^{t}|\dot{\mathcal{E}}|^{\frac{1}{2}} d s\right)^{2} \leq s_{1}^{\frac{1}{2}} \mathcal{E}(0) \text { for } t \leq s_{1}
$$

and on the other hand

$$
\left\|u(t)-u\left(s_{1}\right)\right\|_{L^{2}}^{2} \leq\left\|u(t)-v_{1}(\cdot-c)\right\|_{L^{2}}^{2}+\left\|u\left(s_{1}\right)-v_{1}(\cdot-c)\right\|_{L^{2}}^{2} \lesssim \mathcal{E}_{0} \text { for } t \geq s_{1},
$$


from which we deduce (1.30). Finally, (1.29) follows using Lemma 2.2, the triangle inequality, (2.1), and (1.30):

$$
\begin{aligned}
|\mathbf{x}(t)-\mathbf{x}(0)| & \stackrel{(2.3)}{\lesssim}\|v(t)-v(0)\|_{L^{2}}+\|v(t)-v(0)\|_{L^{2}}^{2} \\
& \stackrel{(2.1)}{\lesssim}\|u(t)-u(0)\|_{L^{2}}+\|u(t)-u(0)\|_{L^{2}}^{2}+\mathcal{E}(0)+\mathcal{E}(0)^{\frac{1}{2}} \\
& \stackrel{(1.30)}{\lesssim} \mathcal{E}(0)+\mathcal{E}(0)^{\frac{1}{2}},
\end{aligned}
$$

where we have allowed $\lesssim$ to depend on $C_{E}, C_{H}$.

\section{Auxiliary Proofs}

\subsection{Preliminary Lemmas}

Proof of Lemma 1.6 For simplicity, let $\bar{x}_{i}=0$. We will show that $u$ has a zero within a neighborhood of $\bar{x}_{i}$ of size $L=C \max \left\{\bar{H}, \bar{H}^{\frac{1}{3}}\right\}$. Note that for any $C>0$ we can choose $\ell_{1}$ so large that

$$
C \max \left\{\bar{H}, \bar{H}^{\frac{1}{3}}\right\} \leq C \max \left\{C_{H}, C_{H}^{\frac{1}{3}}\right\} \leq \ell_{1} .
$$

Assume that $u$ does not have a zero in $(-3 L, 3 L)$. Then $u$ has fixed, say negative, sign on this interval. Let $\bar{v}$ be positive on $\left(0, \bar{x}_{i+1}\right)$. Furthermore, let $\eta: \mathbb{R} \rightarrow[0,1]$ be a cutoff function with

$$
\eta \equiv 1 \text { on }(L, 2 L), \quad \eta \equiv 0 \text { on } \mathbb{R} \backslash(0,3 L)
$$

We estimate

$$
\int_{L}^{2 L} \bar{v} \eta d x \leq \int_{\mathbb{R}}(\bar{v}-u) \eta d x \leq\|u-\bar{v}\|_{L^{2}}\|\eta\|_{L^{2}} \lesssim(\bar{H} L)^{\frac{1}{2}} .
$$

Define $x_{0} \in\left(0, \frac{\bar{x}_{i+1}}{2}\right)$ such that $\bar{v}\left(x_{0}\right)=\frac{1}{2}$. In case $L$ is so small that $x_{0} \in\left[\frac{3}{2} L, \frac{\bar{x}_{i+1}}{2}\right)$, we obtain

$$
\int_{L}^{2 L} \bar{v} \eta d x \geq \int_{L}^{\min \left\{2 L, x_{0}\right\}} \bar{v} d x \gtrsim L^{2}
$$

since $\bar{v} \gtrsim x$ on $\left(0, x_{0}\right)$. If $L$ is large enough so that $x_{0} \in\left(0, \frac{3}{2} L\right)$, on the other hand, we use monotonicity of $\bar{v}$ to estimate

$$
\int_{L}^{2 L} \bar{v} \eta d x \gtrsim L
$$

Combining the previous two estimates with (3.1) yields $L \lesssim \max \left\{\bar{H}, \bar{H}^{\frac{1}{3}}\right\}$, which contradicts the definition of $L$ for $C$ large enough. 
Estimate (1.13) then follows from

$$
\begin{aligned}
\|u-v\|_{L^{2}}^{2} & \lesssim\|u-\bar{v}\|_{L^{2}}^{2}+\|\bar{v}-v\|_{L^{2}}^{2} \\
& \lesssim\|u-\bar{v}\|_{L^{2}}^{2}+|\bar{x}-x|\|\bar{v}-v\|_{L^{\infty}}^{2} \\
& \stackrel{(1.12)}{\lesssim} \bar{H}+\bar{H}^{\frac{1}{3}} .
\end{aligned}
$$

\subsection{Dissipation Proofs from Section 2.1}

To establish Lemma 2.6, we will use comparison with energy minimizers on $\mathbb{R}$ and the following linearized energy gap estimate, which is [20, Lemma 3.12].

Lemma 3.1 For all $f \in H^{1}(\mathbb{R})$ such that

$$
\int f v_{1 x} d x=0, \text { there holds } \int f^{2}+f_{x}^{2} d x \lesssim \int f_{x}^{2}+G^{\prime \prime}\left(v_{1}\right) f^{2} d x .
$$

The proof follows from [19, Lemma 3.1] and bootstrapping as in [17, Proof of Proposition 3.1].

We now use the linearized energy gap to prove Lemma 2.6. The proof is an easy adaptation (simplification) of [20, Proof of Lemma 2.11]. For completeness, we include the details.

Proof of Lemma 2.6 A straightforward calculation leads to

$$
\frac{\mathrm{d}}{\mathrm{d} t} \frac{1}{2} D=-\int g_{x}^{2}+G^{\prime \prime}(u) g^{2} d x
$$

where $g:=u_{x x}-G^{\prime}(u)$, so that it suffices to show

$$
\int g_{x}^{2}+G^{\prime \prime}(u) g^{2} d x \gtrsim-D .
$$

The idea is to use the linearized energy gap estimate on a large interval around zeros of $v$. It suffices to consider one zero; without loss of generality suppose $x_{i}=0$ and recall that the closest neighboring element of $\mathbf{x}$ is at least $\ell \geq \ell_{1}$ away.

We will localize as in [20] using a partition of unity. Assuming that $x_{i}=0$ is neither the smallest nor the largest zero in $\mathbf{x}$, let $\eta: \mathbb{R} \rightarrow[0,1]$ satisfy

$$
\eta \equiv 1 \quad \text { on }\left[\frac{x_{i-1}}{4}, \frac{x_{i+1}}{4}\right], \quad \operatorname{sppt}(\eta) \Subset\left(\frac{3 x_{i-1}}{4}, \frac{3 x_{i+1}}{4}\right), \quad\left|\eta_{x x}\right| \leq \frac{2}{\ell^{2}},
$$

and set $\eta_{-}=(1-\eta) \mathbf{1}_{x \leq 0}, \eta_{+}=(1-\eta) \mathbf{1}_{x \geq 0}$. If $x_{i}=0$ is the smallest zero in $\mathbf{x}$, it suffices to replace $x_{i-1}$ above by $-C$ for a large constant $C \in(0, \infty)$ and analogously if $x_{i}=0$ is the largest element in $\mathbf{x}$.

We claim that

$$
\begin{aligned}
\int g_{x}^{2} d x \geq & \int\left(\left(\eta_{-} g\right)_{x}\right)^{2}+\left((\eta g)_{x}\right)^{2}+\left(\left(\eta_{+} g\right)_{x}\right)^{2} d x \\
& -\frac{2}{\ell^{2}} \int\left(\eta_{-}+\eta+\eta_{+}\right) g^{2} d x, \\
\int G^{\prime \prime}(u) g^{2} d x & \geq \int G^{\prime \prime}(u)\left(\left(\eta_{-} g\right)^{2}+(\eta g)^{2}+\left(\eta_{+} g\right)^{2}\right) d x .
\end{aligned}
$$


The inequality of the integrands in both cases is clear whenever two of the partition functions vanish and it suffices to check the "overlap regions" $I_{-}:=\left[\frac{3 x_{i-1}}{4}, \frac{x_{i-1}}{4}\right]$ and $I_{+}:=\left[\frac{x_{i+1}}{4}, \frac{3 x_{i+1}}{4}\right]$. We choose $\ell_{1}$ sufficiently large and $\varepsilon$ sufficiently small so that

$$
G^{\prime \prime}(u(x)) \geq \frac{1}{C_{1}}>0 \text { for } x \in I_{-} \cup I_{+} .
$$

From this choice and

$$
\eta_{-}^{2}+\eta^{2}+\eta_{+}^{2} \leq 1
$$

we deduce (3.4). For (3.3), we use (3.6), and (3.2), together with the identity

$$
\int\left((\eta g)_{x}\right)^{2} d x=\int \eta^{2} g_{x}^{2}+2 \eta \eta_{x} g g_{x}+\eta_{x}^{2} g^{2} d x=\int \eta^{2} g_{x}^{2}-\eta \eta_{x x} g^{2} d x
$$

for $\eta_{-}, \eta$, and $\eta_{+}$.

We have hence localized the estimates. It suffices to show

$$
\int\left((\eta g)_{x}\right)^{2}+G^{\prime \prime}(u)(\eta g)^{2} d x-\frac{2}{\ell^{2}} \int \eta g^{2} d x \gtrsim-D .
$$

On the support of $\eta$, we project $\eta g$ onto $v_{1 x}$ using

$$
\eta g=g_{0}+\alpha v_{1 x}, \quad \text { with } \alpha:=\frac{\int \eta g v_{1 x} d x}{\int v_{1 x}^{2} d x} .
$$

Notice that by the Cauchy-Schwarz inequality, $|\eta| \leq 1$, and $v_{1 x} \in L^{2}(\mathbb{R})$, there holds

$$
\alpha^{2} \lesssim D
$$

Substituting the decomposition for $\eta g$ and using the equation for $v_{1}$ gives

$$
\begin{aligned}
\int & \left((\eta g)_{x}\right)^{2}+G^{\prime \prime}(u)(\eta g)^{2} d x \\
= & \int\left((\eta g)_{x}\right)^{2}+G^{\prime \prime}\left(v_{1}\right)(\eta g)^{2} d x+\int\left(G^{\prime \prime}(u)-G^{\prime \prime}\left(v_{1}\right)\right)(\eta g)^{2} d x \\
= & \int g_{0 x}^{2}+G^{\prime \prime}\left(v_{1}\right) g_{0}^{2} d x+\int\left(G^{\prime \prime}(u)-G^{\prime \prime}\left(v_{1}\right)\right)(\eta g)^{2} d x .
\end{aligned}
$$

On the one hand, $g_{0} \in H^{1}(\mathbb{R})$ and is orthogonal to $v_{1 x}$, so that we can use the positivity of the energy gap from Lemma 3.1. On the other hand, from the $L^{\infty}$ bound on $u-v$ and the convergence of $v$ to $v_{1}$ with $\ell$, we deduce

$$
\sup _{\left[\frac{3 x_{i-1}}{4}, \frac{3 x_{i+1}}{4}\right]}\left|G^{\prime \prime}(u)-G^{\prime \prime}\left(v_{1}\right)\right| \lesssim \varepsilon+o(1)_{\ell \rightarrow \infty} \leq 2 \varepsilon
$$

for $\ell_{1}$ large enough. Applying these facts yields

$$
\int\left((\eta g)_{x}\right)^{2}+G^{\prime \prime}(u)(\eta g)^{2} d x \gtrsim \int g_{0}^{2}+g_{0 x}^{2} d x-C \varepsilon \int(\eta g)^{2} d x .
$$

According to (3.11), $|\eta| \leq 1$, and Young's inequality, it suffices for (3.7) to show

$$
\int \eta g^{2} d x \lesssim D^{\frac{1}{2}}\left(\int g_{0}^{2} d x\right)^{\frac{1}{2}}+D
$$


This follows easily from

$$
\int \eta g^{2} d x \stackrel{(3.8)}{=} \int \eta g\left(g_{0}+\alpha v_{1 x}\right) d x
$$

together with the Cauchy-Schwarz inequality and (3.9).

We now show that a bound on $\overline{\mathcal{E}}$ induces a bound on $D$.

Proof of Lemma 2.7 For the statement to be nontrivial, we assume that $t \geq s+1$. Using Lemma 1.6 and $|\mathcal{E}-\overline{\mathcal{E}}| \lesssim \exp (-C \ell)$ together with Lemma 2.1, we may assume $\ell_{1}$ and $\gamma$ to be such that (2.17) holds. Let

$$
t_{1}:=\operatorname{argmin}_{[s, s+1]} D, \quad t_{2}:=\operatorname{argmax}_{[s+1, t]} D .
$$

Then on the one hand, the minimum dissipation on $[s, s+1]$ is bounded above via

$$
D\left(t_{1}\right) \leq \int_{s}^{s+1} D(\tau) \mathrm{d} \tau \stackrel{(1.14)}{=} \int_{s}^{s+1}-\frac{\mathrm{d}}{\mathrm{d} t} \overline{\mathcal{E}}(\tau) \mathrm{d} \tau \leq|\overline{\mathcal{E}}(s)|+|\overline{\mathcal{E}}(s+1)| \leq 2 \gamma .
$$

On the other hand, the maximum on $[s+1, t]$ is bounded via

$$
\max _{[s+1, t]} D=D\left(t_{1}\right)+\int_{t_{1}}^{t_{2}} \frac{\mathrm{d}}{\mathrm{d} \tau} D(\tau) \mathrm{d} t \stackrel{(3.12),(2.17)}{\lesssim} \gamma+\int_{t_{1}}^{t_{2}} D(\tau) \mathrm{d} \tau \stackrel{(1.14)}{\lesssim} \gamma .
$$

Proof of Lemma 2.8 Notice that $\mathcal{E} \leq \gamma$ implies $E(u) \lesssim 1$.

The bound on the energy (and the trick of Modica-Mortola) is enough to prevent the existence of zeros that are far from the $N$ zeros in $\mathbf{x}$. Hence, arguing as in the proof of [17, Lemma 3.9], it suffices to show that $\|\xi\|_{L^{\infty}}$ is small on a large neighborhood of any zero of $\mathbf{x}$, where $\xi:=\frac{1}{2} u_{x}^{2}-G(u)$ represents the so-called discrepancy. Indeed, on a neighborhood of any zero $x_{i}, u$ satisfies

$$
u_{x}= \pm \sqrt{2(G(u)+\xi)} \text { with } u\left(x_{i}\right)=0 .
$$

Smallness of the discrepancy hence forces $u$ to be close to a kink profile on a large neighborhood around $x_{i}$ and, in particular, to satisfy

$$
\left|u_{x}\right| \approx \sqrt{2 G(0)} \neq 0 \quad \text { on a large neighborhood of } x_{i},
$$

which rules out nearby zeros.

To see that the discrepancy is small, suppose without loss of generality that $x_{i}=0$ and consider an interval $I=[-L, L]$ for a large, positive $L$. Letting $g:=u_{x x}-G^{\prime}(u)$ we observe

$$
\inf _{I}|\xi| \leq \frac{1}{2 L} \int_{I}|\xi| d x \leq \frac{1}{2 L} \int_{I} \frac{1}{2} u_{x}^{2}+G(u) d x \lesssim \frac{1}{L}
$$

At the same time, there holds

$$
\int\left|\xi_{x}\right| d x \leq \int\left|g u_{x}\right| d x \lesssim D^{\frac{1}{2}} \leq \gamma^{\frac{1}{2}}
$$

The combination of (3.14) and (3.15) implies smallness of the discrepancy on the large interval $I$. 
Acknowledgements Open Access funding provided by Projekt DEAL. It is a pleasure to acknowledge the hospitality of the University of Bonn, where part of this article was written, and financial support from DFG Grant WE 5760/1-1. In addition we thank the anonymous referee for helpful comments.

Open Access This article is licensed under a Creative Commons Attribution 4.0 International License, which permits use, sharing, adaptation, distribution and reproduction in any medium or format, as long as you give appropriate credit to the original author(s) and the source, provide a link to the Creative Commons licence, and indicate if changes were made. The images or other third party material in this article are included in the article's Creative Commons licence, unless indicated otherwise in a credit line to the material. If material is not included in the article's Creative Commons licence and your intended use is not permitted by statutory regulation or exceeds the permitted use, you will need to obtain permission directly from the copyright holder. To view a copy of this licence, visit http://creativecommons.org/licenses/by/4.0/.

\section{Appendix}

Below we include the proof of (2.2) from Lemma 2.1. The steps are very similar to arguments used in $[17,19,20]$. While we give sufficient detail for the arguments to be understood, we refer the interested reader to [17] for an even more detailed view.

Proof of Lemma 2.1, improvement to universal constants and the estimate (2.2) We begin by commenting that (2.1) holds with universal constants once the energy gap falls below $2 e_{*}-\epsilon$. The basic idea is that this condition prevents that $u$ on $\left(x_{i}, x_{i+1}\right)$ (say) is close to a collection of three spread out kinks, which has order-one energy but is far from $v$. This situation is prevented since the solution is bounded away from "the wrong phase" far from elements of $\left\{x_{1}, \ldots, x_{N}\right\}$.

So suppose $\mathcal{E} \leq 2 e_{*}-\epsilon$. Clearly the dependence on $C_{E}$ in (2.1) is removed. For the dependence on $C_{H}$, we argue as follows. Let $I=\left(x_{i}, x_{i+1}\right)$ represent the interval between two elements of $x$ and suppose without loss of generality that $v>0$ on $I$. Choosing $X$ large, we can use the energetic bound to find points $x_{-} \in\left(x_{i}, x_{i}+X\right)$ and $x_{+} \in\left(x_{i+1}-X, x_{i+1}\right)$ such that $u\left(x_{ \pm}\right) \approx 1$. Now we use $\mathcal{E} \leq 2 e_{*}-\epsilon$ and the trick of Modica and Mortola to deduce that there exists $\delta=\delta(\epsilon)$ such that $u \geq-1+\delta$ on $\left(x_{i}+X, x_{i+1}-X\right)$, which in turn implies $G(u) \geq(u-1)^{2} / C_{\delta}$. It follows that

$$
\begin{aligned}
\int_{x_{i}}^{x_{i+1}}(u-v)^{2} d x & =\int_{x_{i}}^{x_{i}+X}(u-v)^{2} d x+\int_{x_{i}+X}^{x_{i+1}-X}(u-v)^{2} d x+\int_{x_{i+1}-X}^{x_{i+1}}(u-v)^{2} d x \\
& \left.\lesssim 1+\int_{x_{i}+X}^{x_{i+1}-X}(u-v)^{2} d x \quad \text { (by the } L^{\infty} \text { bounds on } u \text { and } v\right) \\
& \left.\lesssim 1+\int_{x_{i}+X}^{x_{i+1}-X}(u-1)^{2} d x \quad \text { (by the exponential convergence of } v \text { to } 1\right) \\
& \lesssim 1+C_{\delta} \int_{x_{i}+X}^{x_{i+1}-X} G(u) d x \quad \text { (as explained above) } \\
& \lesssim 1 \quad \text { (by the order-one energetic bound). }
\end{aligned}
$$

The analogous argument applies on the unbounded intervals.

We turn now to the dissipation estimate (2.2). We start with the rough bound: For any $\gamma>0$, if $D \geq \gamma$, then

$$
\|f\|_{H^{1}}^{2} \stackrel{(2.1)}{\leq} C(E(u)-E(v)) \leq \frac{C C_{E}}{\gamma} D .
$$


Hence it suffices to consider the case of small dissipation. In this case we will work locally between two elements of $\mathbf{x}$ (the zeros of $u$ identified in Lemma 1.6) or on $\left(-\infty, x_{1}\right)$ or $\left(x_{N}, \infty\right)$. For notational convenience, we will refer to any such interval as $I$, commenting when necessary on differences for the unbounded intervals. For notational convenience, let

$$
D_{I}=\int_{I}\left(u_{x x}-G^{\prime}(u)\right)^{2} d x .
$$

It suffices to show that for every $C_{H} \in(0, \infty)$ there exists $\gamma>0$ and $C \in(0, \infty)$ such that $D_{I} \leq \gamma$ implies

$$
\|f\|_{H^{1}(I)}^{2} \leq C D_{I} .
$$

We will (i) develop a linear estimate, (ii) show that sup $|u-v|$ is small, (iii) combine those two facts to deduce a nonlinear estimate.

Step $(i)$

We begin with the following linear estimate. There exist $\ell_{1}, C \in(0, \infty)$ such that for $\ell \geq \ell_{1}, v$ as given in the statement of Lemma 2.1, and $f \in H^{1}(I)$ vanishing at both endpoints when $I$ is bounded or one endpoint when $I$ is unbounded, there holds

$$
\|f\|_{H^{1}(I)}^{2} \leq C \int_{I}\left(\mathcal{L}_{A C} f\right)^{2} d x
$$

where we have used the notation $\mathcal{L}_{A C} f=f_{x x}-G^{\prime \prime}(v) f$. This estimate can be deduced from the linear energy gap bound

$$
\|f\|_{H^{1}(I)}^{2} \leq C \int_{I} f_{x}^{2}+G^{\prime \prime}(v) f^{2} d x=C|(f, \mathcal{L} f)|,
$$

as is done in [17, Proof of Proposition 3.1, Equation (3.27)]. Namely, starting with the Cauchy-Schwarz inequality in

$$
\|f\|_{L^{2}(I)}^{2} \leq C|(f, \mathcal{L} f)| \leq C\|f\|_{L^{2}(I)}\|\mathcal{L} f\|_{L^{2}(I)},
$$

we deduce

$$
\|f\|_{L^{2}(I)} \leq C\|\mathcal{L} f\|_{L^{2}(I)}
$$

Now substituting this in the right-hand side of (3.20), we obtain

$$
|(f, \mathcal{L} f)| \leq C\|\mathcal{L} f\|_{L^{2}(I)}^{2} .
$$

Using this in (3.19) returns (3.18) (up to a renaming of $C$ ).

Step (ii)

We will now show that in the case of small dissipation, $f:=u-v$ is small in $L^{\infty}(I)$. For this we argue as in the proof of Lemma 2.8, using the discrepancy $\xi:=\frac{1}{2} u_{x}^{2}-G(u)$ and that on a neighborhood of any zero $x_{i}, u$ satisfies

$$
u_{x}= \pm \sqrt{2(G(u)+\xi)} \text { with } u\left(x_{i}\right)=0 .
$$

Unlike in Lemma 2.8, we do not assume smallness of the energy gap, so that the argument for the smallness of the discrepancy (which proceeds as in the proof of that lemma) yields in this case

$$
\sup _{I}|\xi| \lesssim \frac{C_{E}}{|I|}+C_{E}^{\frac{1}{2}} \gamma^{\frac{1}{2}}
$$


For $\ell_{1}$ large enough and $\gamma$ small enough with respect to $C_{E}$, this gives the desired smallness.

We now invoke an ODE argument. Multiplying the Euler-Lagrange equation with $v_{x}$ and integrating yields that the energy minimizer $v$ satisfies

$$
\begin{cases}v_{x}= \pm \sqrt{2\left(G(v)-G\left(\max _{I} v\right)\right)} & \text { if } I \text { is bounded, } \\ v_{x}= \pm \sqrt{2 G(v)} & \text { if } I \text { is unbounded. }\end{cases}
$$

The combination of (3.21) and (3.22) can be used to deduce that for small enough discrepancy, $u$ has the same sign as and is close to $v$ on large intervals around the zeros $x_{1}, \ldots, x_{N}$; i.e., for any $\varepsilon>0$ and for discrepancy small enough, there holds

$$
\begin{aligned}
& \sup |u-v| \\
& \leq \varepsilon \text { on } \begin{cases}\left(x_{i}, x_{i}+X\right) \cup\left(x_{i+1}-X, x_{i+1}\right) & \text { for } I=\left(x_{i}, x_{i+1}\right) i=1, \ldots, N-1, \\
\left(x_{1}-X, x_{1}\right) & \text { for } I=\left(-\infty, x_{1}\right), \\
\left(x_{N}, x_{N}+X\right) & \text { for } I=\left(x_{N}, \infty\right),\end{cases}
\end{aligned}
$$

for a large, positive constant $X$. On the other hand, using $\|u-v\|_{L^{2}(I)} \leq C_{H}$ and choosing the discrepancy small enough allows us to conclude that $x_{1}, \ldots, x_{N}$ are the only zeros of $u$ and in particular that $u$ has a sign on each subinterval $I$.

It remains only to establish closeness to $v$ on the (large or even unbounded) "middle" of $I$, but this is now easy. To fix ideas, suppose $I=\left(x_{i}, x_{i+1}\right)$ and that $u, v$ are positive on $I$. Then according to the estimate above and the properties of $v, u$ is close to 1 at $x_{N}+X$. But then small discrepancy implies that the the minimum and maximum of $u$ on $\left[x_{i}+X, x_{i+1}-X\right]$ is also close to 1 and, hence, to $v$. The analogous argument applies on the other subintervals, recalling the boundary conditions (cf. Remark 1.4) for the unbounded intervals.

\section{Step (iii)}

Finally, we will combine (i) and (ii) to deduce a nonlinear estimate. As usual let $f:=u-v$. The basic estimate takes the form:

$$
\begin{aligned}
\int_{I}\left(u_{x x}-G^{\prime}(u)\right)^{2} d x & \left.=\int_{I}\left(f_{x x}-G^{\prime}(u)+G^{\prime}(v)\right)^{2} d x \quad \text { (Euler-Lagrange equation of } v\right) \\
& =\int_{I}\left(f_{x x}-G^{\prime \prime}(v) f-G^{\prime}(u)+G^{\prime}(v)+G^{\prime \prime}(v) f\right)^{2} d x \\
& \geq \frac{1}{2} \int_{I}\left(\mathcal{L}_{A C}\right)^{2} d x-C\|f\|_{L^{\infty}(I)}^{2} \int_{I} f^{2} d x .
\end{aligned}
$$

Applying $\|f\|_{L^{\infty}(I)} \leq \varepsilon$ (for $\varepsilon>0$ sufficiently small) and the linear estimate (3.18) is enough to conclude (3.17). Notice that $\gamma$, and hence the constant in (3.16), depends on both $C_{E}$ and $C_{H}$.

The fact that the constants in the dissipation estimate are universal in the case of small energy gap is not hard to check, since the dependence on $C_{E}$ is replaced with a universal constant and (2.1) implies $\|u-v\|_{L^{2}} \lesssim 1$ as well.

\section{References}

1. Alikakos, N., Bates, P.W., Fusco, G.: Slow motion for the Cahn-Hilliard equation in one space dimension. J. Differ. Equ. 90(1), 81-135 (1991)

2. Argentina, M., Clerc, M.G., Rojas, R., Tirapegui, E.: Coarsening dynamics of the one-dimensional CahnHilliard model. Phys. Rev. E 71(4), 046210 (2005) 
3. Bates, P.W., Xun, J.P.: Metastable patterns for the Cahn-Hilliard equation. I. J. Differ. Equ. 111(2), 421-457 (1994)

4. Bates, P.W., Xun, J.P.: Metastable patterns for the Cahn-Hilliard equation. II. Layer dynamics and slow invariant manifold. J. Differ. Equ. 117(1), 165-216 (1995)

5. Brézis, H.: Opérateurs Maximaux Monotones et Semi-groupes de Contractions Dans les Espaces de Hilbert. North-Holland Publishing Co., Amsterdam-London; American Elsevier Publishing Co., Inc., New York, (1973) (French). North-Holland Mathematics Studies, No. 5. Notas de Matemática (50)

6. Bricmont, J., Kupiainen, A., Taskinen, J.: Stability of Cahn-Hilliard fronts. Commun. Pure Appl. Math. 52(7), 839-871 (1999)

7. Bronsard, L., Hilhorst, D.: On the slow dynamics for the Cahn-Hilliard equation in one space dimension. Proc. R. Soc. Lond. Ser. A 439(1907), 669-682 (1992)

8. Bronsard, L., Kohn, R.V.: On the slowness of phase boundary motion in one space dimension. Commun. Pure Appl. Math. 43(8), 983-997 (1990)

9. Carlen, E.A., Carvalho, M.C., Orlandi, E.: A simple proof of stability of fronts for the Cahn-Hilliard equation. Commun. Math. Phys. 224(1), 323-340 (2001). Dedicated to Joel L. Lebowitz

10. Carr, J., Pego, R.L.: Metastable patterns in solutions of $u_{t}=\epsilon^{2} u_{x x}-f(u)$. Commun. Pure Appl. Math. 42(5), 523-576 (1989)

11. Chen, X.: Generation, propagation, and annihilation of metastable patterns. J. Differ. Equ. 206(2), 399437 (2004)

12. Eckmann, J.-P., Rougemont, J.: Coarsening by Ginzburg-Landau dynamics. Commun. Math. Phys. 199(2), 441-470 (1998)

13. Elliott, C.M., French, D.A.: Numerical studies of the Cahn-Hilliard equation for phase separation: IMA. J. Appl. Math. 38(2), 97-128 (1987)

14. Fusco, G., Hale, J.K.: Slow-motion manifolds, dormant instability, and singular perturbations. J. Dyn. Differ. Equ. 1(1), 75-94 (1989)

15. Grant, C.P.: Slow motion in one-dimensional Cahn-Morral systems. SIAM J. Math. Anal. 26(1), 21-34 (1995)

16. Howard, P.: Asymptotic behavior near transition fronts for equations of generalized Cahn-Hilliard form. Commun. Math. Phys. 269(3), 765-808 (2007)

17. Otto, F., Reznikoff, M.G.: Slow motion of gradient flows. J. Differ. Equ. 237(2), 372-420 (2007)

18. Otto, F., Scholtes, S., Westdickenberg, M.G.: Optimal $L^{1}$-type relaxation rates for the Cahn-Hilliard equation on the line. SIAM J. Math. Anal. 51(6), 4645-4682 (2019)

19. Otto, F., Westdickenberg, M.G.: Relaxation to equilibrium in the one-dimensional Cahn-Hilliard equation. SIAM J. Math. Anal. 46(1), 720-756 (2014)

20. Scholtes, S., Westdickenberg, M.G.: Metastability of the Cahn-Hilliard equation in one space dimension. J. Differ. Equ. 265(4), 1528-1575 (2018)

21. Sun, X., Ward, M.J.: Dynamics and coarsening of interfaces for the viscous Cahn-Hilliard equation in one spatial dimension. Stud. Appl. Math. 105(3), 203-234 (2000)

22. Ward, M.J.: Metastable patterns, layer collapses, and coarsening for a one-dimensional Ginzburg-Landau equation. Stud. Appl. Math. 91(1), 51-93 (1994)

Publisher's Note Springer Nature remains neutral with regard to jurisdictional claims in published maps and institutional affiliations. 